\title{
An AXL/LRP-1/RANBP9 complex mediates DC efferocytosis and antigen cross-presentation in vivo
}

\author{
Manikandan Subramanian, ${ }^{1}$ Crystal D. Hayes, ${ }^{2}$ Joseph J. Thome, ${ }^{3,4}$ Edward Thorp, ${ }^{5}$ \\ Glenn K. Matsushima, ${ }^{6}$ Joachim Herz,7 Donna L. Farber, ${ }^{3,4}$ Kang Liu, ${ }^{4}$ \\ Madepalli Lakshmana, ${ }^{2}$ and Ira Tabas ${ }^{1,8}$
}

\begin{abstract}
'Department of Medicine, Columbia University, New York, New York, USA. ${ }^{2}$ Torrey Pines Institute for Molecular Studies, Port Saint Lucie, Florida, USA ${ }^{3}$ Columbia Center for Translational Immunology and ${ }^{4}$ Department of Microbiology and Immunology, Columbia University, New York, New York, USA. 5Department of Pathology and FCVRI, Northwestern University, Chicago, Illinois, USA. 'Department of Microbiology and Immunology, UNC Neuroscience Center, University of North Carolina at Chapel Hill, Chapel Hill, North Carolina, USA. ${ }^{7}$ Departments of Molecular Genetics, Neuroscience, Neurology, and Neurotherapeutics, University of Texas Southwestern Medical Center, Dallas, Texas, USA.

${ }^{8}$ Department of Pathology and Cell Biology and Department of Physiology and Cellular Biophysics, Columbia University, New York, New York, USA.
\end{abstract}

\begin{abstract}
The phagocytosis of apoptotic cells (ACs), or efferocytosis, by DCs is critical for self-tolerance and host defense. Although many efferocytosis-associated receptors have been described in vitro, the functionality of these receptors in vivo has not been explored in depth. Using a spleen efferocytosis assay and targeted genetic deletion in mice, we identified a multiprotein complex - composed of the receptor tyrosine kinase AXL, LDL receptorrelated protein-1 (LRP-1), and RAN-binding protein 9 (RANBP9) - that mediates DC efferocytosis and antigen cross-presentation. We found that AXL bound ACs, but required LRP-1 to trigger internalization, in murine CD8 $\alpha^{+}$DCs and human-derived DCs. AXL and LRP-1 did not interact directly, but relied on RANBP9, which bound both AXL and LRP-1, to form the complex. In a coculture model of antigen presentation, the AXL/ LRP-1/RANBP9 complex was used by DCs to cross-present AC-associated antigens to $T$ cells. Furthermore, in a murine model of herpes simplex virus-1 infection, mice lacking DC-specific LRP-1, AXL, or RANBP9 had increased $\mathrm{AC}$ accumulation, defective viral antigen-specific $\mathrm{CD8}^{+} \mathrm{T}$ cell activation, enhanced viral load, and decreased survival. The discovery of this multiprotein complex that mediates functionally important DC efferocytosis in vivo may have implications for future studies related to host defense and DC-based vaccines.
\end{abstract}

\section{Introduction}

Efferocytosis is the process by which apoptotic cells (ACs) are recognized and cleared by phagocytic cells $(1,2)$. The crucial role of this process is exemplified by the development of spontaneous autoimmunity secondary to breakdown of self-tolerance in mice lacking certain efferocytosis receptors or their bridging molecules (3-6). Whereas macrophages are highly efficient at AC uptake (7) and are usually the focus of efferocytosis studies, efferocytosis by DCs can mediate the presentation of AC-associated antigens on $\mathrm{MHC}$ class I to naive and memory $\mathrm{CD}^{+} \mathrm{T}$ cells in a process called cross-presentation. This process has been proposed to induce either antigenic tolerance (8) or T cell priming against infectious agents (9), depending on the specific context. Mostly in vitro studies have identified a number of distinct efferocytosis receptors that can be used by DCs $(7,10,11)$, suggestive of possible redundancy or tissue- or context-specific usage. However, the identity and mechanisms of receptors utilized by DCs to perform functionally important efferocytosis and cross-presentation in vivo constitutes a major gap in this important area of research.

Previous reports showed that the efferocytosis receptors CD36, $\alpha v \beta 5$, and $\alpha v \beta 3$ are not involved in antigen cross-presentation by DCs $(12,13)$. Bone marrow-derived DCs (BMDCs) from the AXL/ TYRO3 double-KO mouse demonstrate defective efferocytosis in vitro (11), but the role of AXL/TYRO3 in DC efferocytosis in

Conflict of interest: The authors have declared that no conflict of interest exists. Citation for this article: J Clin Invest. 2014;124(3):1296-1308. doi:10.1172/JCI72051. vivo and in cross-presentation is not known. An important study showed that $\mathrm{T}$ cell Ig domain and mucin domain 3 (TIM-3) participates in AC uptake by DCs and cross-presents a model antigen OVA to OT-I transgenic T cells (10). However, the relative contribution of TIM-3 in the context of other DC receptors and in functionally relevant cross-presentation is not known, especially because TIM-3 is also a negative regulator of T cell activation (14). Recently, scavenger receptor class F, member 1 (SCARF-1), was reported to be important for efferocytosis by DCs, and SCARF-1deficient mice developed a lupus-like autoimmune disease (15). However, whether SCARF-1 plays a role in antigen cross-presentation or is utilized by human DCs was not explored. Given the critical role of DC efferocytosis and cross-presentation in maintaining tissue homeostasis, peripheral tolerance, and elicitation of antiviral immunity upon infection and vaccination, identification of the mechanisms and functionality of DC efferocytosis receptors in vivo is of paramount importance.

To address this critical gap in DC biology, we first used an in vivo spleen efferocytosis assay in which mice with absent or inhibited candidate DC efferocytosis receptors were injected with labeled ACs, followed by analysis of AC uptake by splenic DCs. Based on these initial findings, we carried out molecular-cellular studies and then gene-targeted mouse experiments to interrogate mechanism and function. This investigation led to the identification of a multiprotein efferocytosis complex that mediates $\mathrm{DC}$ efferocytosis in vivo. The proteins in the complex, AXL, LDL receptor-related protein-1 (LRP-1; also known as CD91), and 


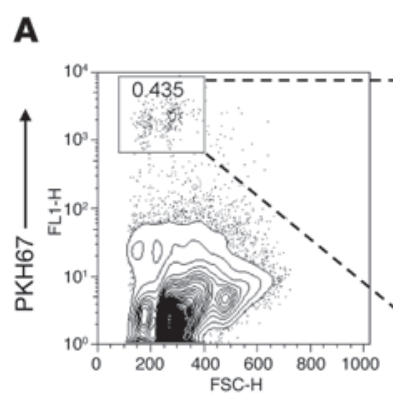

B Control Gate: $\mathrm{PKH} 67^{+}$cells

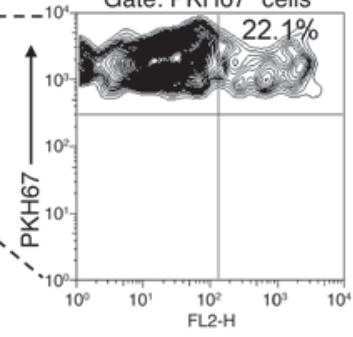

$\mathrm{CD} 11 \mathrm{c}$

C
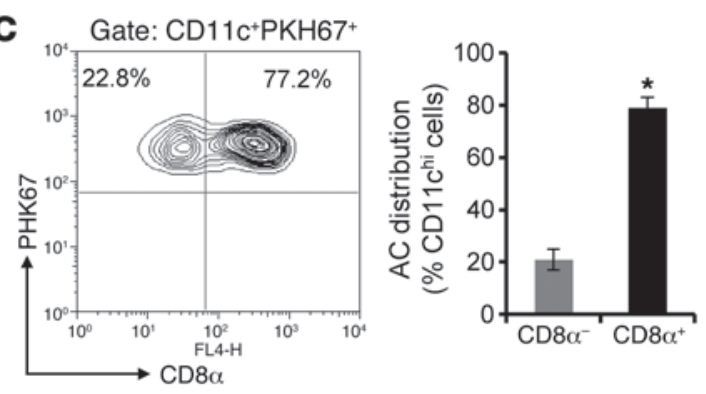

$\mathrm{CpG}$

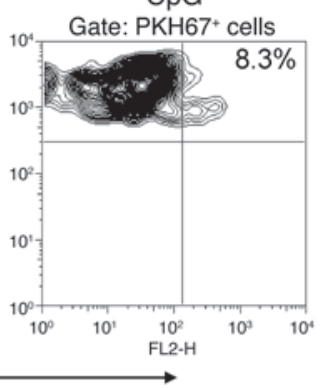

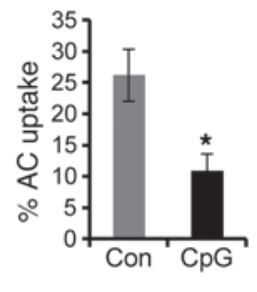

D
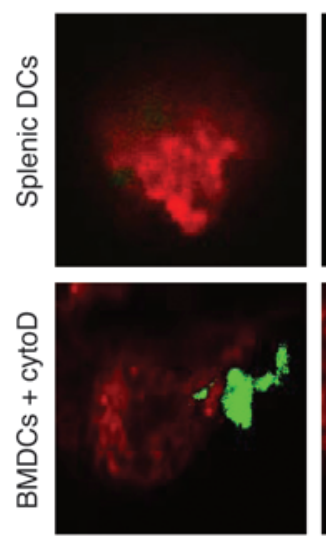
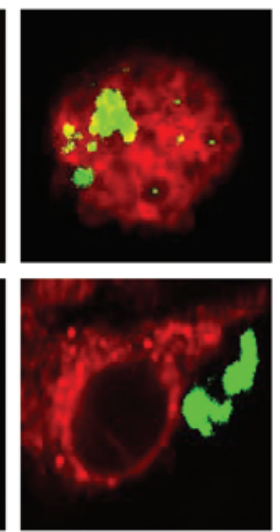
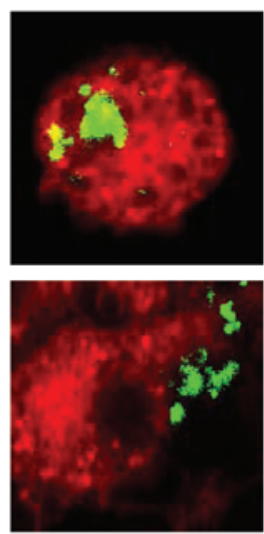

E

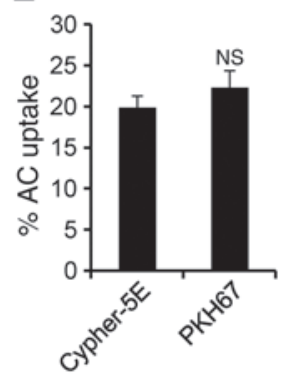

\section{Figure 1}

In vivo splenic DC efferocytosis assay. (A) Flow cytometric detection of cells that costained for PKH67 (square gate) in the splenocytes of mice injected with PKH67-labeled ACs. (B) Analysis and quantification ( $n=3$ per group) of the AC-gated region in $\mathbf{A}$ to determine the proportion of ACs that were internalized by splenic DCs (CD11 $\left.\mathrm{C}^{+}\right)$in C57BL/6J mice injected with vehicle control (Con) or the DC maturation stimulus CpG-DOTAP. Numbers represent the percentage of cells in the respective quadrants. (C) Flow cytometric analysis and quantification ( $n=3$ mice per group) of the distribution of internalized ACs between the CD8 $\alpha^{+}$and CD8 $\alpha^{-}$subsets of CD11 $\mathrm{C}^{+} \mathrm{PKH} 67^{+}$-gated splenocytes. (D) Top: Confocal microscopy $z$ section sequence (top-to-bottom at 2- $\mu$ m intervals) of FACS-sorted CD11 $\mathrm{C}^{+} \mathrm{PKH} 67^{+}$splenic DCs (representative of cells in the top-right quadrant of B), demonstrated the presence of ACs inside the DCs. Red, PE staining of splenic CD11c ${ }^{+}$cells; green, PKH67-labeled ACs. Bottom: $z$ section sequence of BMDCs (red) treated with $5 \mu \mathrm{g} / \mathrm{ml}$ cytochalasin D (CytoD) prior to addition of ACs (green) to demonstrate what a DC would look if there was AC binding, but not engulfment. Scale bars: $5 \mu \mathrm{m}$. (E) Comparison of in vivo splenic DC efferocytosis in mice injected with ACs labeled with PKH67 or Cypher-5E ( $n=3$ per group). ${ }^{*} P<0.05$ vs. respective control.

RAN-binding protein 9 (RANBP9), were unable to effect efferocytosis on their own, but rather functionally interacted to coordinate AC binding and internalization. Most importantly, these molecules played a substantial, nonredundant role in the crosspresentation of AC-associated antigens in vivo, including in clearance of a viral infection. Human splenic and monocyte-derived DCs also used these molecules for efferocytosis. Knowledge of the presence and functionality of this multiprotein complex in DC efferocytosis may have implications for future studies related to host defense and DC-based vaccines.

\section{Results}

Splenic DCs utilize AXL, LRP-1, and TIM-3 for clearance of ACs in vivo and in vitro. As a first approach to interrogating DC efferocytosis in vivo, we injected PKH67-labeled ACs into mice and then quantified the uptake of those ACs by splenic DCs using flow cytometry (16). To help validate this assay, we took advantage of the fact that mature splenic DCs, which can be increased in mice by injection with the Toll-like receptor activator CpG-DOTAP, and CD8 $\alpha^{-}$ splenic DCs have low efferocytosis efficiency $(7,17)$. The assay successfully identified DCs from CpG-treated mice and CD8 $\alpha^{-}$DCs 
A
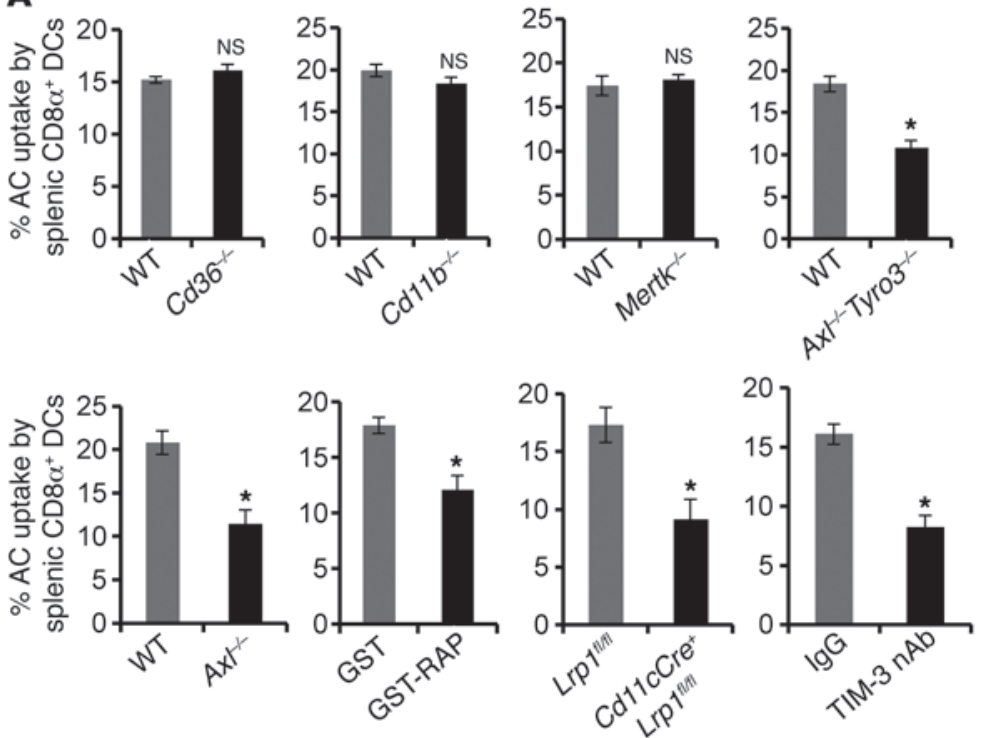

B

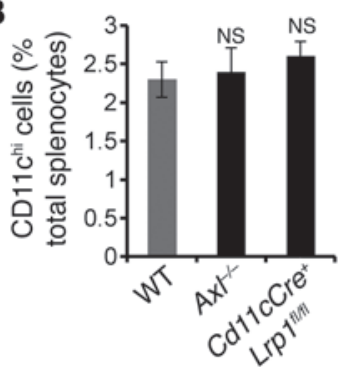

C

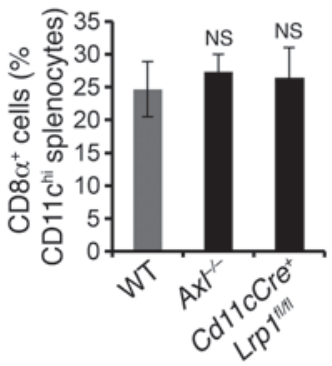

D

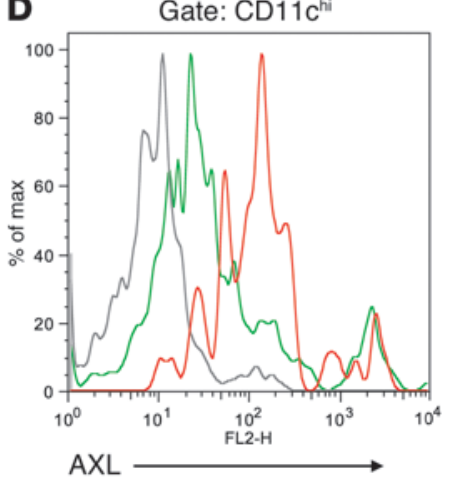

E

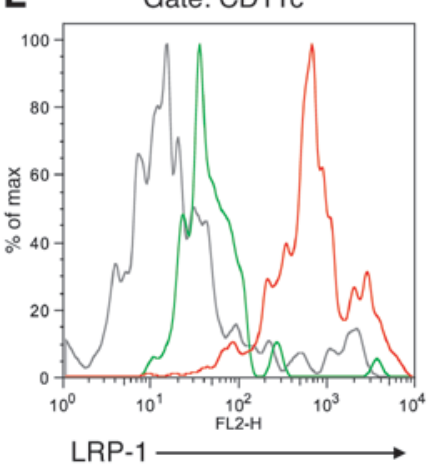

$\mathbf{F}$

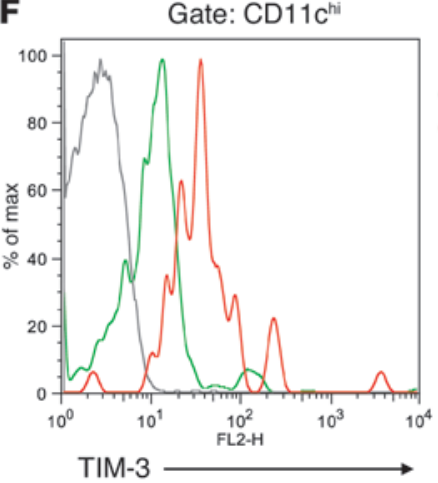

$\operatorname{lgG}$ control CD $8 \alpha^{-}$DC $\mathrm{CD} 8 \alpha^{+} \mathrm{DC}$

Figure 2

Screening for DC efferocytosis receptors in vivo. (A) In vivo splenic DC efferocytosis assay conducted 3 hours after injection of ACs in control mice and mice with absent or inhibited candidate efferocytosis receptors ( $n=3$ per group). The $y$ axis represents the proportion of ACs engulfed by splenic CD8 $\alpha^{+}$DCs. LRP-1 was inhibited by i.v. injection of GST-RAP (2 mg/mouse), and TIM-3 was inhibited by i.p. injection of an anti-TIM-3 nAb (RMT3-23; $100 \mu \mathrm{g} /$ mouse). (B and C) Flow cytometric quantification of the percentage of total DCs (CD11 $\mathrm{c}^{\text {hi }}$ ) and CD8 $\alpha^{+}$DCs in the spleens of the indicated mice ( $n=3$ per group). (D-F) Flow cytometric analysis of expression of AXL (D), LRP-1 (E), and TIM-3 (F) in CD8 $\alpha^{+}$and CD8 $\alpha^{-}$ splenic DCs of WT mice. ${ }^{*} P<0.05$ vs. respective control.

as poor efferocytes compared with DCs from untreated mice and CD8 $\alpha^{+}$DCs, respectively (Figure 1, A-C). Furthermore, FACS sorting and confocal microscopic analysis of $\mathrm{CD} 11 \mathrm{c}^{+} \mathrm{PKH} 67^{+}$splenocytes revealed that more than $97 \%$ of these cells showed evidence of AC internalization. A representative image demonstrating internalization of ACs in these sorted cells $\left(\mathrm{CD} 11 \mathrm{c}^{+}\right.$gate; Figure $\left.1 \mathrm{~B}\right)$ is shown in Figure 1D. As an example of what noninternalization of ACs would look like by confocal microscopy, BMDCs were treated with the actin polymerization inhibitor cytochalasin $\mathrm{D}$ prior to addition of ACs. The $z$ section sequence in this case showed that ACs were present only at the cell surface (Figure 1D). To further demonstrate internalization, we showed that efferocytosis in vivo was similar using either PKH67-labeled ACs or ACs labeled with the $\mathrm{pH}$-sensitive dye Cypher-5E (Figure 1E), which fluoresces only after entering the acidic late endosomes or lysosomes.

Application of this assay to mice with absent or inhibited candidate receptors revealed that deficiency of CD36, CD11b, or MERTK did not affect efferocytosis by CD8 $\alpha^{+}$splenic DCs (Figure 2A). A previous study using BMDCs from AXL/TYRO3 double-KO mice demonstrated a role for one or both of these receptors in DC efferocytosis in vitro (11). Here, we found that not only $A x^{-/-}$ Tyro3 $^{-1-}$ mice, but also $A x l^{l^{--}}$mice, were defective in DC efferocytosis in vivo (Figure $2 \mathrm{~A}$ ), establishing that AXL is a physiologically important DC efferocytosis receptor.

We next explored LRP-1, which has previously been implicated in efferocytosis by macrophages, but not DCs $(18,19)$. Inhibition of LRP-1 by i.v. injection of GST-conjugated receptor-associated protein (GST-RAP), which binds LRP-1 and inhibits interaction of the receptor with a number of LRP-1 ligands (Supplemental Figure 1A; supplemental material available online with this article; doi:10.1172/JCI72051DS1; and ref. 20), inhibited splenic DC efferocytosis by approximately 50\% (Figure 2A). To confirm the involvement of LRP-1 in DC efferocytosis, we generated Cd11cCre $\operatorname{Lrp} 1^{f / f l}$ mice, which have undetectable LRP-1 in splenic 
A

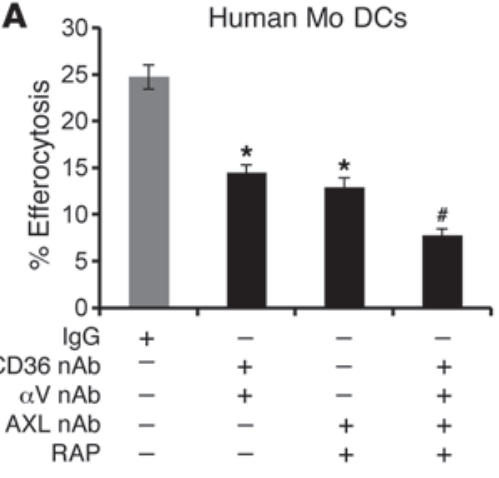

C

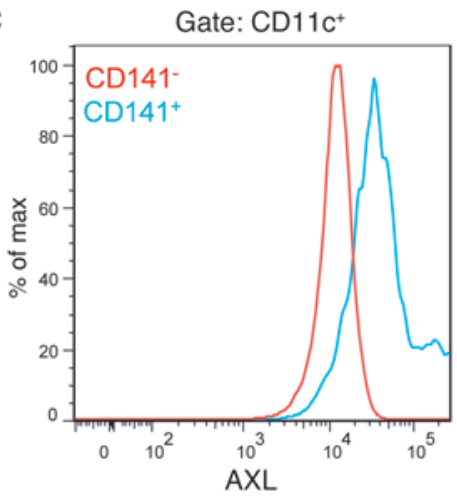

B
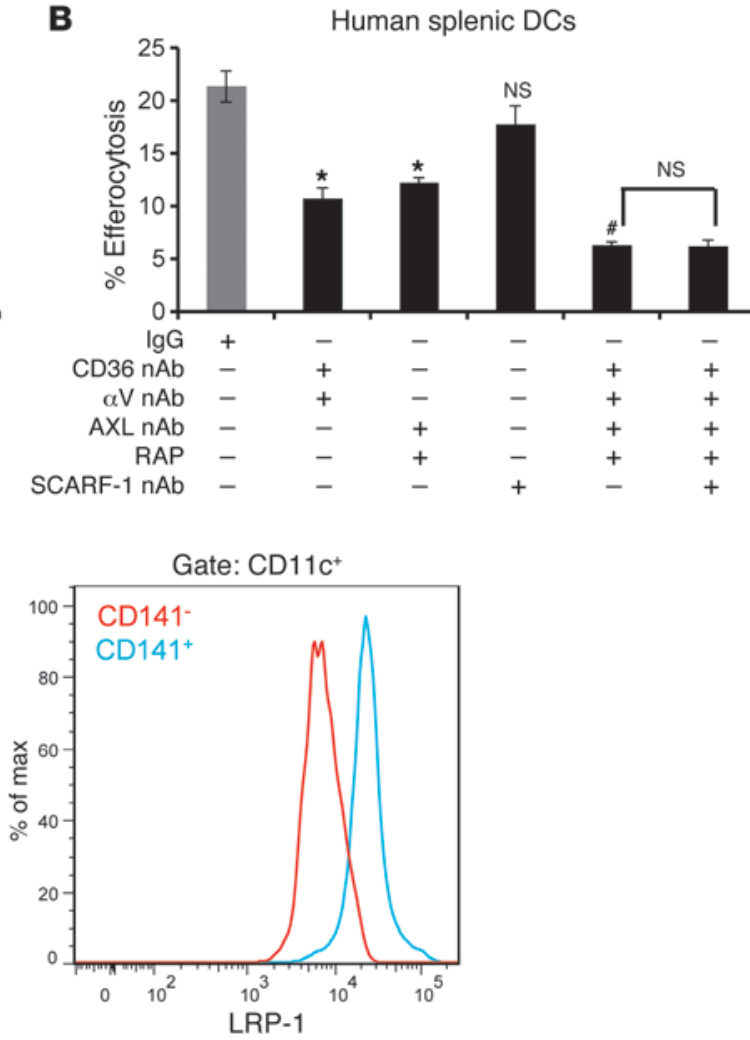

\section{Figure 3}

Human monocyte-derived DCs and splenic DCs utilize AXL and LRP-1 for efferocytosis. (A and $\mathbf{B}$ ) Human monocyte-derived DCs (MoDCs; $n=5 ; \mathbf{A})$ and splenic DCs $(n=7 ; \mathbf{B})$ were incubated with IgG control or the indicated combinations of nAbs against $\mathrm{AXL}, \mathrm{CD} 36, \alpha \mathrm{V}$, or SCARF-1 $(0.5 \mu \mathrm{g} / \mathrm{ml})(R \& D$ Systems) or the LRP-1 blocker GST-RAP ( $1 \mu \mathrm{g} / \mathrm{ml})$. Cells were then exposed to PKH67labeled apoptotic Jurkat cells $(1: 4$ ratio) for 2 hours and assayed for efferocytosis by microscopic analysis. (C) Representative flow cytometric analysis of expression levels of $A X L$ and LRP-1 in human CD141- and CD141+ splenic DCs $(n=5) .{ }^{*} P<0.05$ vs. respective control; $\#>0.05$ vs. all other groups (except as indicated).
DCs but intact expression in splenic macrophages (Supplemental Figure 1B). CD8 $\alpha^{+}$splenic DCs in these mice showed significantly decreased efferocytosis compared with control $\operatorname{Lrp} 1^{f l / f l}$ mice (Figure $2 \mathrm{~A}$ ), further validating a role for LRP-1 in DC efferocytosis in vivo. Further studies showed that $\mathrm{CD} 8 \alpha^{+} \mathrm{DCs}$, which had greater efferocytic efficiency than CD8 $\alpha^{-}$DCs (Figure 1C), expressed substantially higher levels of AXL and LRP-1 compared with CD8 $\alpha^{-}$ DCs (Figure 2, D and E); this was also the case for another DC efferocytosis molecule, TIM-3 (Figure $2 \mathrm{~F}$ and see below). In this context, the decrease in efferocytosis observed in the $A x l^{-/-}$and $C d 11 c C r e^{+} \operatorname{Lrp}^{f l / f l}$ mice was not due to changes in the number of total or CD8 $\alpha^{+}$DCs (Figure 2, B and C) or in the efferocytosis efficiency of splenic macrophages (Supplemental Figure 2). Furthermore, the spatial distribution of DCs within the splenic marginal zone, where most of the injected ACs traffic (21), was not different among WT, $A x l^{l^{-}-}$, and Cd11cCre ${ }^{+} \operatorname{crp} 1^{f l / f l}$ mice (data not shown).

We next explored 2 other molecules previously implicated in DC efferocytosis. Consistent with a previous report (10), injection of anti-TIM-3 neutralizing antibody (nAb) decreased efferocytosis by DCs in vivo (Figure 2A). An nAb against SCARF-1, another DC molecule recently shown to mediate the uptake of ACs (15), also inhibited efferocytosis by BMDCs in vitro in a manner that was additive to AXL and TIM-3 (Supplemental Figure 3A). Finally, we tested which molecules mediate efferocytosis by human splenic DCs and monocyte-derived DCs. Neutralization of AXL and LRP-1 using anti-AXL antibody and GST-RAP, respectively, was associated with a significant reduction in human DC efferocytosis (Figure 3, A and $\mathrm{B}$ ). The degree of inhibition was comparable to that obtained by neutralization of CD36 and $\alpha v \beta 3 / \beta 5$, which were previously reported to facilitate efferocytosis by human DCs (7). Importantly, simultaneous blockage of all the receptors resulted in an additive effect on efferocytosis inhibition (Figure 3, A and B). However, blocking SCARF-1 using an nAb did not decrease efferocytosis by human splenic DCs, either singly or in combination with other efferocytosis receptor blocking antibodies (Figure 3B). To demonstrate that the anti-SCARF-1 nAb was functional in human splenic DCs, we showed that it markedly decreased the uptake of one of SCARF-1's ligands, acetylated LDL (Ac-LDL; Supplemental Figure 3B). Interestingly, AXL and LRP-1 were highly expressed on human CD $141^{+}$splenic DCs (Figure 3C), which are orthologous to murine $\mathrm{CD} 8 \alpha^{+} \mathrm{DCs}$ in their efferocytosis and cross-presentation abilities $(22,23)$. Thus, similar to our observations with AXL/LRP-1 and TIM-3 in mice, human monocyte-derived DCs and splenic DCs used AXL/LRP-1, but not SCARF-1, in conjunction with other, parallel receptor pathways to carry out efferocytosis.

$A X L$ and LRP-1 cooperate to mediate efferocytosis. In view of our finding that the decreased DC efferocytosis in the spleens of $\mathrm{Axl}^{l^{-/}}$, $C d 11 c C r e^{+} L r p 1^{f / f l}$, and TIM-3-neutralized mice was only partial, we next sought to determine whether DC efferocytosis can be blocked further by various combinations of receptor inhibition. When $A \times l^{-1-}$ mice were treated with an anti-TIM-3 nAb, the inhibition of splenic DC efferocytosis was additive (Figure 4A). This was not the case for CD36 or $\alpha \mathrm{V}$ integrins (Supplemental Figure 4A), further supporting the data in Figure 2A suggesting these molecules are not involved in efferocytosis by murine DCs. Importantly, treatment of $\mathrm{Axl}^{\mathrm{H}^{-}}$mice with the LRP-1 inhibitor GST-RAP did not result in any additional decrease in efferocytosis compared with each manipulation alone (Figure 4B), which suggests that LRP-1 and AXL function in the same pathway. In this context, it is important to note that expression levels of LRP-1 in Axl $\mathrm{l}^{-/}$DCs and AXL in LRP-1-deficient DCs were comparable to those in WT DCs (Supplemental Figure 4B). 

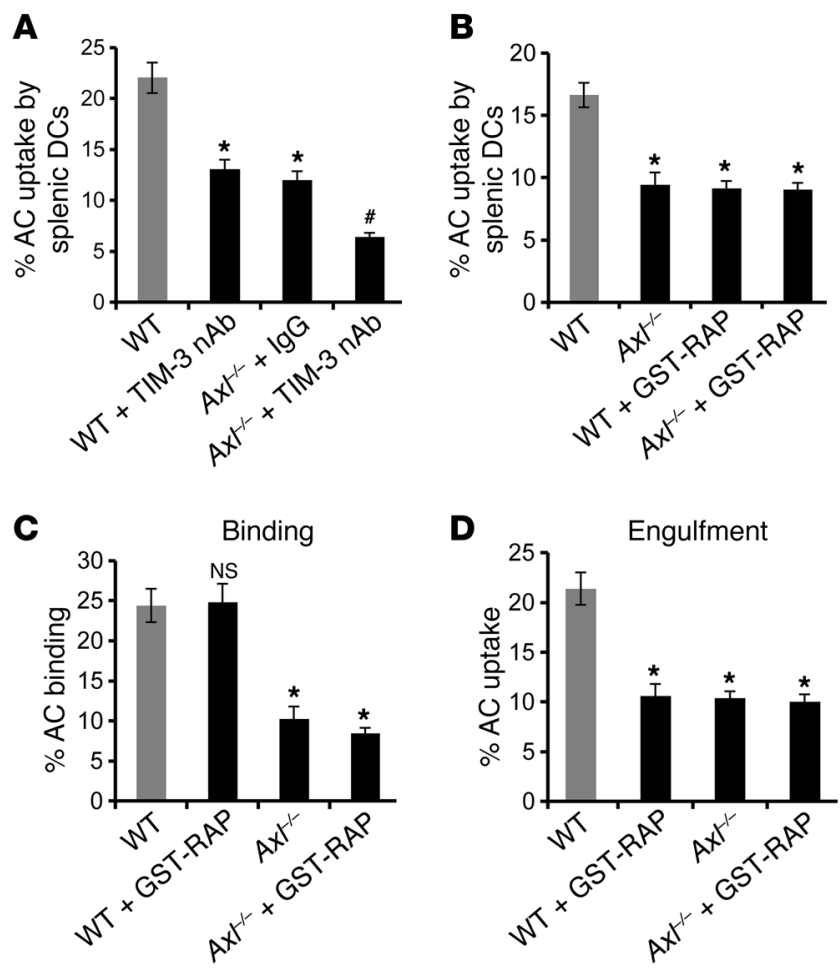

To further explore the concept that AXL and LRP-1 might function in the same pathway, we considered the possibility that LRP-1 and AXL might carry out different steps in efferocytosis, such as $\mathrm{AC}$ recognition versus engulfment (24). To assess the role of AXL and LRP-1 in AC binding, we used the actin polymerization inhibitor cytochalasin D to prevent AC internalization. Whereas $A x l^{-/-}$DCs were clearly deficient in AC binding using this assay, inhibiting LRP-1 using GST-RAP did not affect this process (Figure $4 \mathrm{C}$ ). In contrast, when the assay was carried out in the absence of cytochalasin D to enable engulfment, both AXL deficiency and GST-RAP were inhibitory (Figure 4D). Similar results were obtained when the binding experiment was conducted at $4^{\circ} \mathrm{C}$ to block internalization (data not shown). Notably, blocking LRP-1 in $A \times l^{-/-}$BMDCs did not further augment the defect in AC engulfment (Figure 4D), consistent with the in vivo data in Figure 4B. These findings suggest a model in which AXL is involved primarily in AC recognition by DCs, whereas LRP-1 functions in engulfment of the bound ACs, but not AC binding per se.

RANBP9 facilitates the functional interaction of AXL and LRP-1 in efferocytosis. We reasoned that if AXL and LRP-1 functioned in a binding-internalization cooperative manner, they might be in close proximity or even physically interact with each other. To test this hypothesis, we first conducted double immunofluorescence confocal microscopy on BMDCs. Within the resolution of this technique, the images were suggestive of AXL/LRP-1 colocalization on the cell surface (Figure 5A). We also found that LRP-1 was expressed at a higher level than AXL in DCs (data not shown), which likely explains our finding that percent colocalization of AXL with LRP-1 was higher than that of LRP-1 with AXL (78\% vs. $41 \%$ ). To test our hypothesis using a complementary method, we determined whether AXL and LRP-1 could be coimmunoprecipitated in BMDCs. co-IP experiments showed the presence of LRP-1 in AXL IPs and the presence of AXL in LRP-1 IPs (Figure

\section{Figure 4}

AXL and LRP-1 cooperate to facilitate DC efferocytosis. (A and $\mathbf{B}$ ) In vivo splenic $\mathrm{DC}$ efferocytosis was assayed in the indicated mice ( $n=3$ per group) 3 hours after injection of PKH67-labeled ACs. TIM-3 nAb $(100 \mu \mathrm{g})$ was administered i.p., and GST-RAP (2 mg/mouse) was administered i.v., 30 minutes before AC injection. (C) BMDCs derived from WT or $A \mathrm{xl}^{-1-}$ mice were treated with $5 \mu \mathrm{g} / \mathrm{ml}$ cytochalasin $D$ to block $A C$ internalization. To measure AC binding, cells were incubated with fluorescently labeled ACs for 1 hour in the absence or presence of $1 \mu \mathrm{g} / \mathrm{ml}$ GST-RAP to block LRP-1. (D) Similar to C, except the experiment was conducted in the absence of cytochalasin $D$ to measure AC engulfment. $n=3$ independent experiments. ${ }^{*} P<0.05$ vs. respective control; $\# P<0.05$ vs. all other groups.

5B). Notably, AXL was not detected in the IPs of LRP-1 in $A x^{-/-}$ DCs (Supplemental Figure 5A), confirming the specificity of the antibodies used in these experiments. To determine whether AXL and LRP-1 interact in splenic DCs in vivo, we conducted a fluorescence resonance energy transfer (FRET) experiment in which primary antibodies recognizing AXL and LRP-1 were conjugated to Alexa Fluor 546 or Alexa Fluor 647 to act as FRET donor and acceptor, respectively. This assay was based on the principle that photons emitted by donor fluorophore can excite acceptor fluorophore when they are within $100 \AA$ of each other. A positive FRET is indicated by both an increase in fluorescence of the acceptor (sensitized emission) and a concomitant decrease in fluorescence of the donor (donor fluorescence quenching). FACS analysis of splenic DCs showed substantial FRET, as measured by analysis of both sensitized emission and donor fluorescence quenching (Figure 5, $\mathrm{C}$ and D). Moreover, consistent with our confocal data demonstrating greater colocalization of AXL with LRP-1, donor fluorescence quenching was greater when antibody against AXL was used as a donor (Figure 5D). As a control for this experiment, we showed that FRET was not observed in $A x^{-/-}$and LRP-1-deficient splenic DCs (Supplemental Figure 5B), thereby validating the fluorophore-conjugated antibodies used in this assay. In addition, FRET between fluorophore-conjugated antibodies against heavy and light chains of MHC class I, which are known to interact on the cell surface, was used as a positive control (Supplemental Figure 5C).

In order to determine the functional significance of AXL/LRP-1 interaction, it was necessary to identify the mechanism responsible for their association. Protein interaction algorithms suggested that LRP-1 and AXL do not directly bind each other, so we hypothesized the presence of a scaffolding protein. Further database searching indicated that the protein RANBP9 interacts with AXL in cancer cells (25) and with LRP-1 in neuronal cells (26). Although RANBP9 has not previously been implicated in any functional interaction between AXL and LRP-1 or in efferocytosis, we considered the hypothesis that it can act as an AXL/LRP-1 scaffolding protein in DCs to couple AXL-mediated AC binding with LRP-1-mediated AC internalization.

We first probed the LRP-1 and AXL IPs for RANBP9 and found a positive immunoblot signal in both cases (Figure 5B), consistent with our scaffolding hypothesis. To test the functional significance of this interaction, we created bone marrow chimeric mice

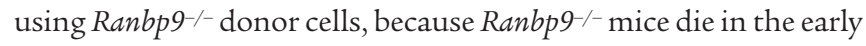
postnatal period (27). Thus, fetal liver cells from E15 Ranbp $9^{--}$and WT embryos were transplanted into lethally irradiated C57BL/6J mice to generate chimeric mice in which BMDCs were deficient in RANBP9. As confirmation, we verified that splenic DCs in the 
A
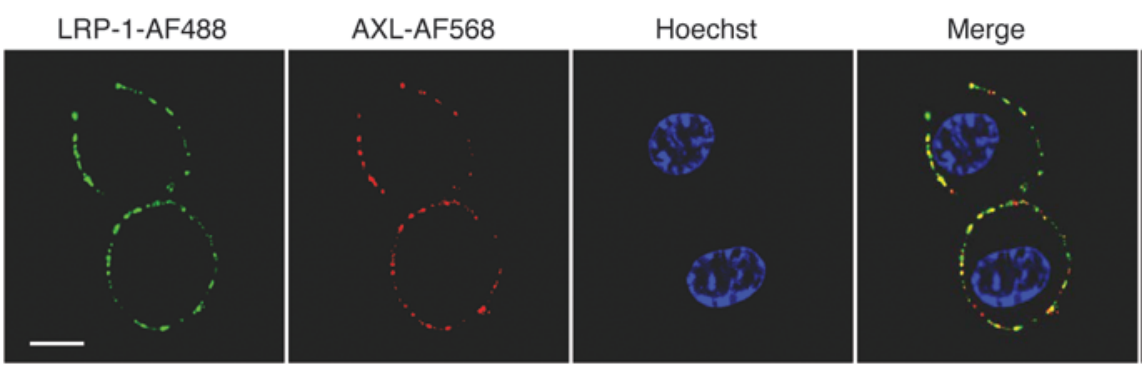

Colocalization

B

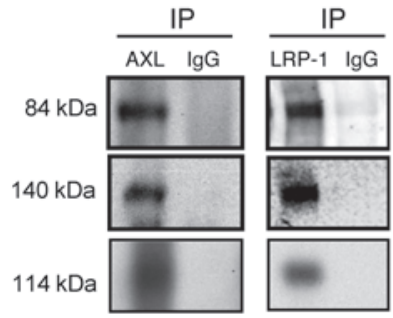

WB: LRP-1

WB: AXL

WB: RANBP9

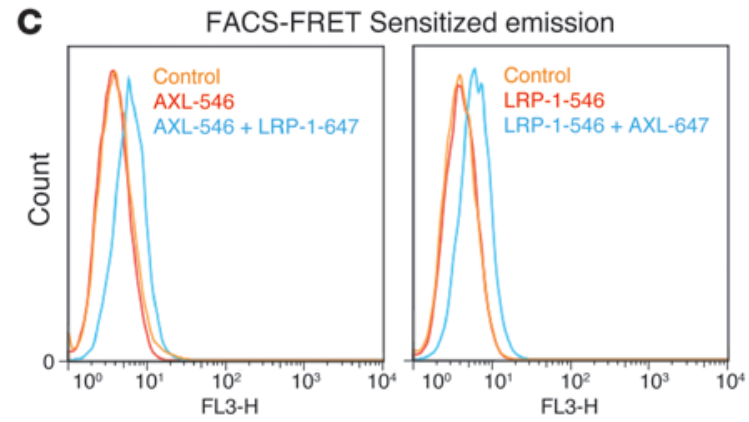

D

FACS-FRET Donor fluorescence quenching
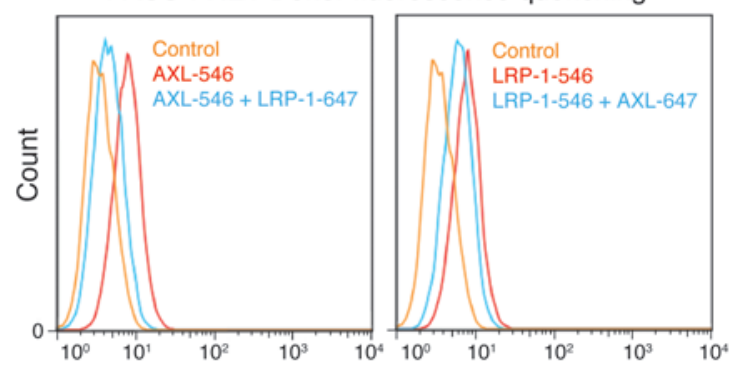

\section{Figure 5}

AXL and LRP-1 interact in DCs in vitro and in vivo. (A) Representative immunofluorescent confocal microscopy of BMDCs stained at $4{ }^{\circ} \mathrm{C}$ with anti-LRP-1 antibody followed by Alexa Fluor 488-labeled secondary antibody (green), and with anti-AXL antibody followed by Alexa Fluor 568labeled secondary antibody (red). Points of AXL and LRP-1 colocalization are indicated by yellow staining in the merged image as well as in the mask of colocalization. Scale bar: $5 \mu \mathrm{m}$. (B) Endogenous AXL or LRP-1 was subjected to IP from whole-cell lysates of BMDCs, then probed by Western blotting for AXL, LRP-1, or RANBP9. Rabbit or rat IgG in the IP reaction was used as control. (C) Flow cytometric analysis of FRET (sensitized emission) in splenic DCs (CD11 $c^{+}$gate) immunostained with fluorophore-conjugated antibody against AXL as donor and LRP-1 as acceptor (left) or vice versa (right). Other cells were stained singly with fluorophore-conjugated antibody against AXL (left) or LRP-1 (right) as donor. Cells stained with IgG conjugated to Alexa Fluor 546 were used as controls. (D) Donor fluorescence measurements in cells as in C. Data are representative of 3 independent experiments.

chimeric mice lost expression of RANBP9 compared with WT DCs (Supplemental Figure 6A). However, neither the expression level of AXL and LRP-1 in DCs nor the total number of CD8 $\alpha^{+}$ DCs was affected by RANBP9 deficiency (Supplemental Figure 6, B-D). Consistent with our hypothesis, RANBP9 deficiency in DCs (assessed using BMDCs from Ranbp 9 fl/fl $^{f}$ mice transduced with adeno-Cre) resulted in loss of FRET signal between AXL and LRP-1 (Figure 6A). Moreover, AXL failed to coimmunoprecipitate with LRP-1 in Ranbp9 $9^{-1-}$ DCs (Figure 6B). These data demonstrated that RANBP9 deficiency disrupted AXL/LRP-1 interaction. Most importantly, efferocytosis by splenic DCs was defective in Ranbp9-/chimeric mice (Figure 6C), but efferocytosis by splenic macrophages was unaffected (Supplemental Figure 6E). Furthermore, consistent with the concept that AXL functions to bind ACs, while AXL/LRP-1 interaction is necessary for subsequent engulfment, BMDCs from Ranbp9-/- chimeric mice showed normal AC binding, but defective AC engulfment (Figure 6D).
$\mathrm{AC}$ internalization after recognition by specific efferocytosis receptors involves actin cytoskeletal reorganization mediated by the GTP-bound form of Rac1 (28). Previous reports indicated that LRP-1 mediates AC engulfment through its adapter protein, engulfment adaptor PTB domain containing 1 (GULP1) (29), which facilitates the formation of Rac-GTP (30). Consistent with our hypothesis that LRP-1/GULP1 is primarily involved in engulfment of AXL-bound ACs, silencing GULP1 using siRNA in WT BMDCs (knockdown efficiency, >80\%; data not shown) led to a significant decrease in AC engulfment, but AC binding was unaffected (Figure 6E). Furthermore, silencing GULP1 in $A x^{-/-}$ BMDCs did not result in an additional decrease in AC engulfment compared with $A x l^{l^{-}-}$BMDCs transfected with negative control scrambled RNA (Supplemental Figure 7), which suggests that LRP-1/GULP1 by itself is unable to mediate AC recognition and internalization. These data support the concept that AXL and LRP-1/GULP1 function as a complex in which AXL binds 
A

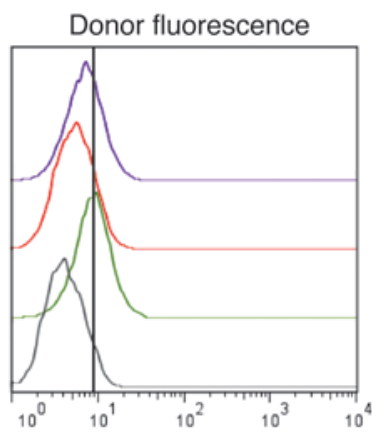

FACS-FRET

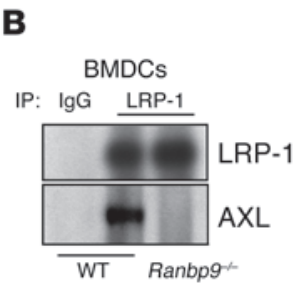

E

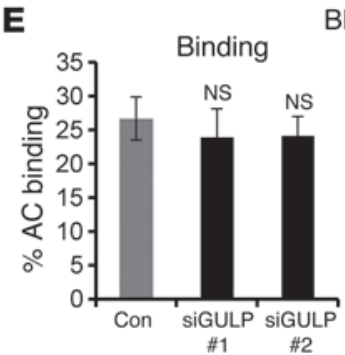

Sensitized emission

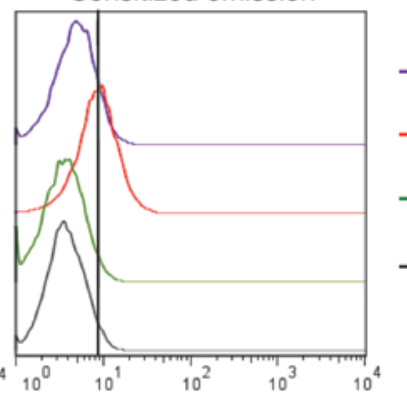

BMDCs

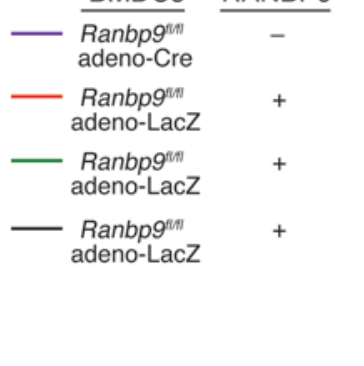

Alexa Ab

AXL-546 LRP-1-647

AXL-546

LRP-1-647

AXL-546

IgG-546

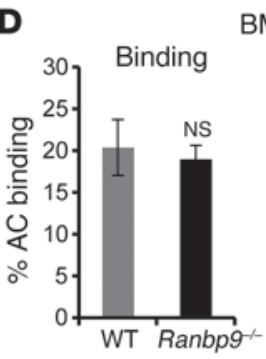

BMDCs

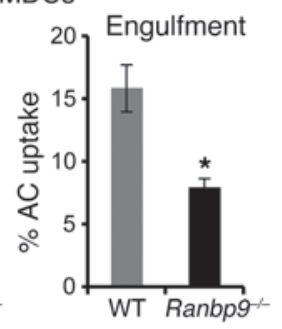

ference in the uptake of DiI-labeled LDL, a measure of receptor-mediated endocytosis (Figure 7B). Similarly, there was no difference in the ability of these DCs to phagocytose fluorescently labeled $1-\mu \mathrm{m}$ latex beads (Figure 7C). Thus, AXL- and LRP-1-deficient DCs have a specific defect in efferocytosis.

The AXL/LRP-1/RANBP9 efferocytosis receptor complex is critical for cross-presentation of AC-derived antigens, both in vitro and in vivo. As mentioned above, one of the functional implications of DC efferocytosis is its ability to utilize AC-derived antigens for cross-presentation. We therefore tested whether ACs internalized via the AXL/LRP-1/ RANBP9 complex could provide antigens for cross-presentation. We first used an in vitro model in which DCs from WT, $A x l^{-/-}$, or Cd11cCre ${ }^{+}$Lrp $^{\prime f l f l}$ mice were incubated with OVA-loaded ACs and then cocultured with CFSE-labeled $\mathrm{CD}^{+}$OT-I transgenic T cells. Both $A x l^{-/-}$and $C d 11 c C r e^{+} L r p 1^{f l / f l}$ DCs demonstrated decreased activation and proliferation of OT-I transgenic $T$ cells (Figure 8A), indicative of defective antigen cross-presentation in the mutant DCs. Importantly, the $A x l^{-/-}$and $C d 11 c C r e^{+} \operatorname{Lrp} 1^{f / f l}$ DCs were not intrinsically defective at cross-presentation, since they were robust in cross-presenting soluble as well as bead-conjugated OVA (Figure 8, B and C).

The OVA assays were conducted in vitro using a model antigen, which is not directly relevant in vivo under physiologic or pathologic conditions. We therefore tested whether efferocytosis via the AXL/ LRP-1/RANBP9 complex has relevance in antigen cross-presentation in a natural virus infection model. To achieve this, we used an experimental model of systemic herpes

ACs and LRP-1/GULP1 mediates AC engulfment through actin cytoskeletal rearrangement.

AXL- and LRP-1-deficient DCs do not have defects in macropinocytosis and phagocytosis. As a test of specificity for the role of the AXL/ LRP-1/RANBP9 complex in DC efferocytosis, we next determined whether these molecules also play roles in the related processes of macropinocytosis, endocytosis, and phagocytosis. BMDCs obtained from WT, $A x l^{-/}$, and $C d 11 c C r e^{+} \operatorname{Lrp} 1^{f / / f /}$ mice did not demonstrate any difference in the uptake of FITC-labeled dextran, a measure of macropinocytosis (Figure 7A). There was also no dif- simplex virus-1 (HSV-1) infection in mice, because clearance of HSV- 1 in vivo is dependent on successful cross-presentation and elicitation of a protective $\mathrm{CD}^{+} \mathrm{T}$ cell response (31). To monitor cross-presentation and activation of antigen-specific $\mathrm{CD}^{+}$ $\mathrm{T}$ cells, we used mice that were adoptively transferred with CFSElabeled $\mathrm{CD}^{+} \mathrm{T}$ cells isolated from gBT-I.1 transgenic mice. These $\mathrm{T}$ cells are MHC class I-restricted, with antigen specificity to the immunodominant peptide from HSV glycoprotein B (gB498505) (32). Mice lacking AXL, LRP-1 in DCs (Cd11cCre+Lrp1 $\left.1^{f l f f}\right)$, or RANBP9 in BMDCs had a significant increase in the number 

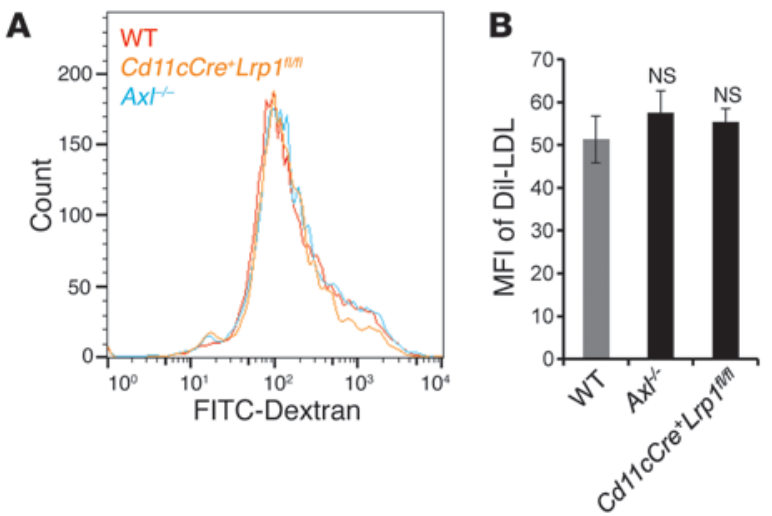

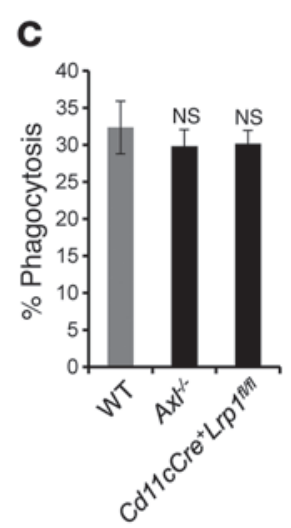

\section{Figure 7}

Macropinocytosis, receptor-mediated endocytosis, and phagocytosis are not altered in BMDCs lacking $A X L$ or LRP-1. BMDCs from the indicated groups of mice were assayed for $(\mathbf{A})$ macropinocytosis of FITClabeled dextran, (B) receptor-mediated endocytosis of Dil-labeled LDL, and (C) phagocytosis of $1-\mu \mathrm{m}$ fluorescent beads $(n=3)$. of nonproliferating gB498-505-specific $\mathrm{CD}^{+} \mathrm{T}$ cells in HSV-1infected spleens (Figure 9A and Supplemental Figure 8A). Most importantly, in light of the role of $\mathrm{CD}^{+} \mathrm{T}$ cells in anti-HSV-1 immunity, all $3 \mathrm{KO}$ models showed enhanced virus-induced pathogenesis, as demonstrated by adrenomegaly (Supplemental Figure 8B), massive adrenal infiltration of monocytes and neutrophils (data not shown), and viral titers more than 3-fold higher than in control mice (Figure 9B). Moreover, the adrenal glands of the mutant mice showed a significant increase in the number of TUNEL $^{+}$cells (Figure 9C), consistent with both increased HSV-1induced apoptosis (33) and decreased efferocytic clearance of ACs. In theory, these data could be influenced by increased permissiveness of the KO cells to HSV-1 infection; however, HSV-1 infectivity was not increased in BMDCs lacking AXL or LRP-1 or in primary adrenal cells lacking AXL (Supplemental Figure 9). Furthermore, to directly relate these data to efferocytosis of HSV-1-killed cells, we rendered primary adrenal cells apoptotic by ex vivo infection with HSV-1 and then injected these ACs into WT and mutant mice. Consistent with the interpretation of the HSV-1 infection data, $A x l^{l^{-}-}$and $C d 11 c C r e^{+} L r p 1^{f / f l}$ mice demonstrated defective DC efferocytosis and decreased cross-presentation to gBT-I.1 CD8 ${ }^{+}$ T cells (Supplemental Figure 10, A and B). Finally, to confirm and extend the biological significance of these findings, we compared the survival of WT and $C d 11 c C r e^{+} L r p 1^{f l / f l}$ mice infected with a lethal dose of HSV-1 and found significantly decreased median survival in $C d 11 c C r e^{+} \operatorname{Lrp} 1^{f l / f l}$ compared with WT mice (Cd11cCre $\operatorname{Lrp}^{+1 / f l}, 5.5$ days; WT, 8.5 days; Figure 9D). Thus, despite the presence of multiple DC efferocytosis receptors, the AXL/LRP-1/RANBP9 efferocytosis complex was functionally important in antigen acquisition for cross-presentation and elicitation of a protective $\mathrm{CD}^{+}$cytotoxic $\mathrm{T}$ cell response in the setting of a natural virus infection.

A recent in vitro study demonstrated that enveloped viruses utilize DC TYRO3/AXL/MERTK (TAM) family receptors, including AXL, to promote viral replication via suppression of type I IFN responses (34). However, our in vivo data demonstrated that $A \mathrm{xl}^{-/-}$ mice were more susceptible to HSV-1 (an enveloped virus) compared with WT mice on days 4, 7, and 10 after infection (Figure 9B and Supplemental Figure 11A). Moreover, the levels of IFN- $\alpha$ in serum were not significantly different between WT and $A x l^{-/-}$mice (Supplemental Figure 11B). These data raise the interesting possibility that the pathogen and host may compete for the same cellular machinery. For example, during initial phases of infection, viruses may utilize TAM receptors on DCs to suppress an early IFN- $\alpha$ response and promote viral replication, while during later stages of infection, particularly when virus-induced ACs arise, DCs utilize the TAM receptor AXL to recognize ACs, including virus-infected ACs, triggering activation of $\mathrm{CD}^{+}$cytotoxic $\mathrm{T}$ cell response and clearance of virus.

\section{Discussion}

Efferocytosis by DCs serves several important functions, including maintenance of tolerance to self-antigens; initiation of immune response against virally infected cells; and, in theory, prevention of secondary necrosis of uncleared ACs (8). Numerous receptors have been implicated through in vitro and occasionally in vivo studies as being able to mediate AC uptake by a variety of phagocytic cell types (35). While these studies suggest that different types of phagocytes preferentially utilize specific sets of receptors to carry

\section{Figure 8}

AXL- and LRP-1-deficient DCs show defective crosspresentation of antigens only acquired by efferocytosis. BMDCs from the indicated mice were pulsed with antigens by incubating with OVA-loaded apoptotic Jurkat cells (A), soluble OVA (B), or bead-conjugated OVA (C), then coincubated with CFSE-labeled CD8 ${ }^{+}$ OT-I T cells for 72 hours. OT-I T cell proliferation was measured by flow cytometric analysis of CFSE dye dilution in $\mathrm{CD}^{+}{ }^{+}$-gated cells. Graphs are representative of 2 independent experiments. ${ }^{*} P<0.05$ vs. respective control.
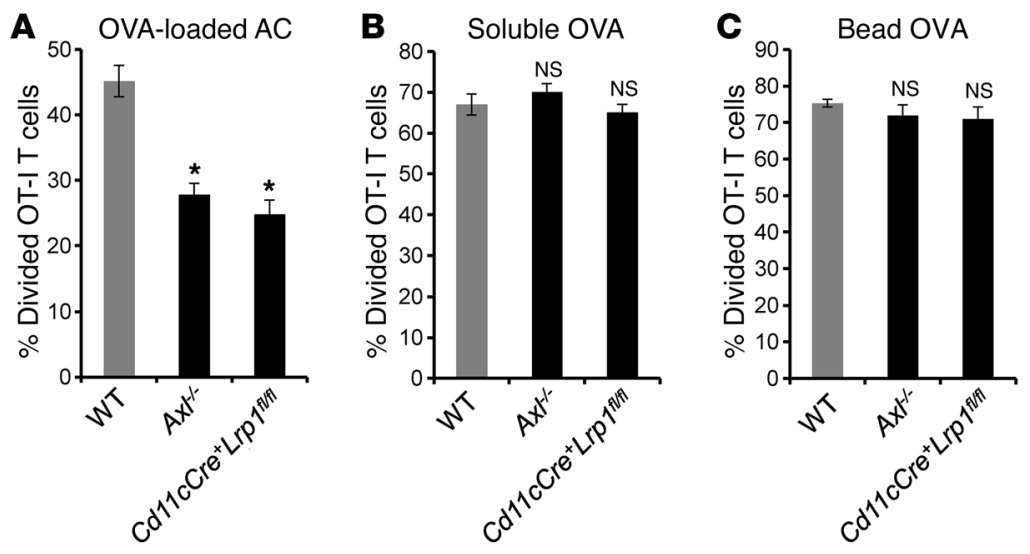
A

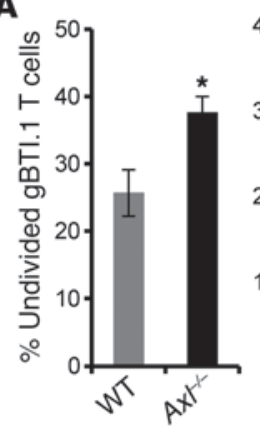

C
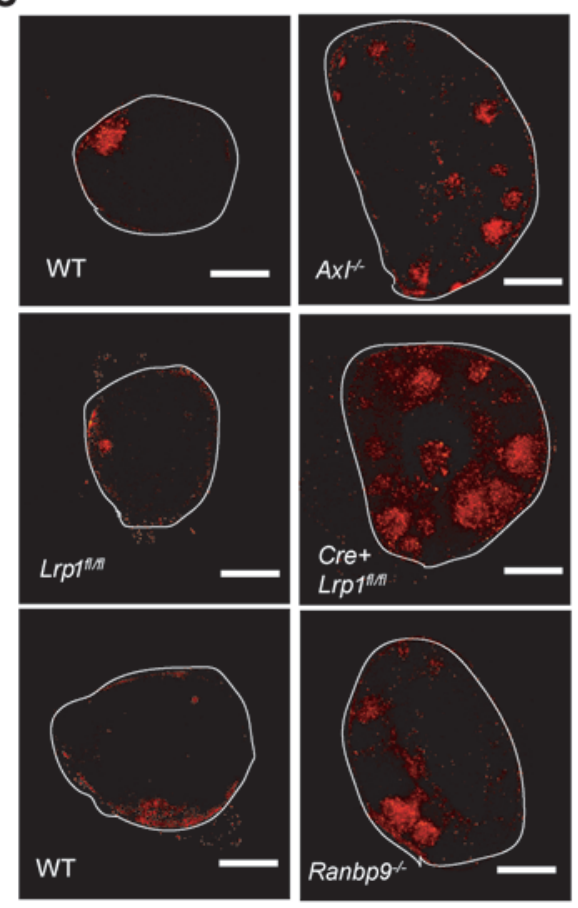

\section{B}

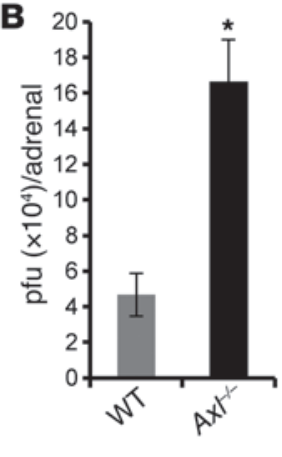

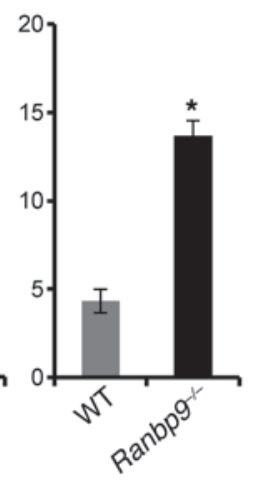
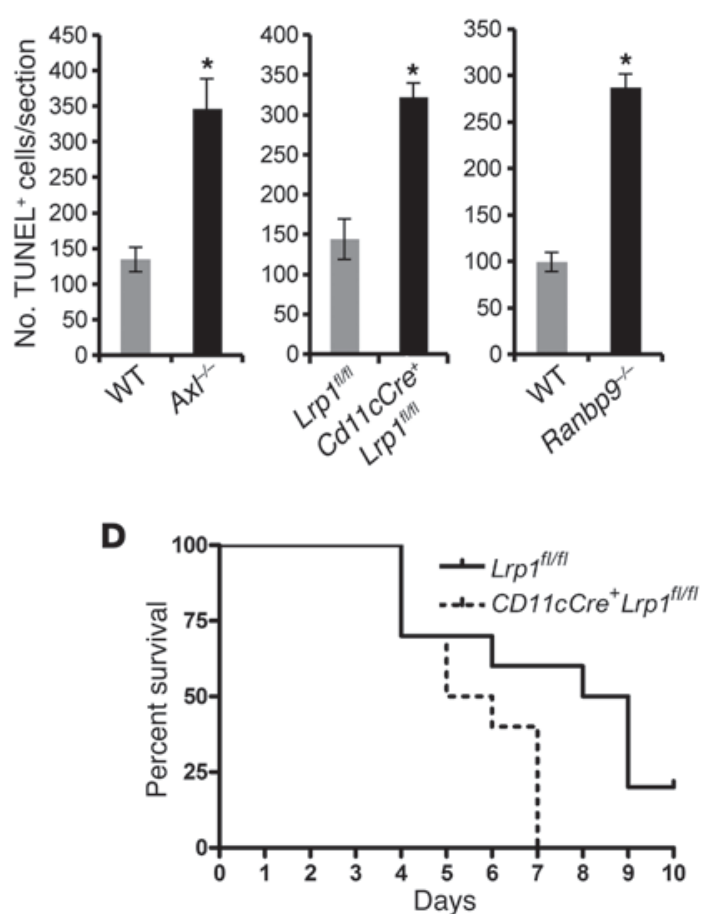

\section{Figure 9}

RANBP9, AXL, and LRP-1 play important roles in cross-presentation in an HSV-1 infection model. (A) Mice were infected i.v. with HSV-1 strain $\mathrm{KOS}\left(4 \times 10^{5} \mathrm{pfu} / \mathrm{mouse}\right)$. All mice also received $10^{6} \mathrm{CFSE}-$ labeled CD8 ${ }^{+} \mathrm{T}$ cells purified from spleens of gBT-I. 1 transgenic mice. 4 days after infection, spleens of infected mice were analyzed for transgenic T cell proliferation by flow cytometric analysis of CFSE ${ }^{+} \mathrm{CD} 8^{+}$gated T cells. Data show percent undivided CFSE-labeled gBT-I.1 transgenic T cells. (B) HSV-1 titers in mouse adrenal glands 4 days after infection with HSV-1. (C) Representative microscopic images and quantification ( $n=4$ mice per group) of TUNEL staining in mouse adrenal glands 4 days after infection with HSV-1. Scale bars: $500 \mu \mathrm{m}$. (D) Kaplan-Meier survival curve analysis of WT Lrp $1^{\mathrm{fl} / \mathrm{fl}}$ mice and Cd11cCre+Lrp $1^{f / l f l}$ mice infected with a lethal dose of HSV-1 $\left(2 \times 10^{8} \mathrm{pfu} / \mathrm{mouse}\right)$. Median survival of WT mice was 8.5 days, compared with 5.5 days for Cd11cCre+Lrp1 $1^{\mathrm{fl} / \mathrm{fl}}$ mice $(P<0.05$; $n=10$ per group). ${ }^{*} P<0.05$ vs. respective control.

out efferocytosis, there has been considerable uncertainty as to the receptors used by DCs for functionally important efferocytosis in vivo. The findings of the present study help clarify this critical area, with implications for understanding the roles of DC efferocytosis in physiology, pathophysiology, and possibly therapy, such as in DC-vaccine design (36).

AXL interacts with ACs through the bridging molecule GAS6, which binds both AXL and phosphatidylserine exposed on the surface of ACs (37). However, the mechanism by which AXL mediates AC engulfment was not previously elucidated. Here, we demonstrated that in murine DCs, AXL primarily acted as an
AC recognition receptor that bound ACs, but did not internalize them. Engulfment of the bound ACs required RANBP9-mediated interaction of AXL with the LRP-1/GULP1 module. This mode of action raises the possibility of the tether-and-tickle hypothesis proposed by Henson and colleagues, in which macrophages were shown to bind ACs through one of a number of receptors, but internalization required another molecule, referred to as PSR (24). In DCs, AXL is an AC tether, and it is possible that this process somehow "tickles" the LRP-1/GULP complex to mediate cytoskeletal rearrangement and AC internalization. However, further studies will be needed to define if and how AC binding trig- 
gers activation of the LRP-1/GULP complex, including whether RANBP9 simply keeps AXL and LRP-1 close enough to allow a direct tickle-type interaction between AXL and LRP-1 or whether RANBP9 plays a more complex role in the actual transmission of the putative tickle signal.

Several lines of in vitro evidence suggest that antigens derived from ACs via efferocytosis are cross-presented by DCs to activate antigen-specific $\mathrm{CD}^{+} \mathrm{T}$ cells $(9,38,39)$. Antigen cross-presentation is an incompletely understood process that involves transfer of antigen-derived peptides into the cytosolic or vacuolar pathway for loading onto MHC class I (40). This process is influenced by a number of factors, including efficiency of antigen capture, nature of antigen, dose, expression levels of MHC class I, and antigen processing via proteolysis, which in turn can be regulated by the kinetics of endosomal maturation and acidification (40). Specifically, retention of the antigenic material in a less acidic early endosomal-like compartment appears to be critical for efficient antigen cross-presentation. Consistent with this idea, antigens targeted to different endocytosis receptors that vary in their kinetics of lysosomal targeting are cross-presented with different efficiencies (41). In this context, it is interesting to note that while CD36, $\alpha v \beta 5$, and $\alpha v \beta 3$ mediate uptake of ACs by human DCs in vitro, they do not play a significant role in antigen cross-presentation in vitro (12, 13). Thus, the identification of efferocytosis receptors that are efficient at targeting antigens for cross-presentation will likely have important implications for understanding basic DC biology and for therapeutically targeting antigens to optimally activate $\mathrm{CD}^{+}$ $\mathrm{T}$ cells. Our present data showed that the AXL/LRP-1/RANBP9 complex in DCs mediated cross-presentation of AC-derived antigens both in vitro in an OVA-OT-I model as well as in vivo in a natural HSV-1 infection model. Consistent with a previous report, blocking TIM-3 using an anti-TIM-3 nAb resulted in inhibition of both efferocytosis and cross-presentation of AC-associated antigen in vitro (data not shown). However, in view of the key roles of TIM-3 in T cell biology (14), addressing the role of TIM-3 in crosspresentation in vivo will require the use of a DC-specific TIM-3 deletion model, which has not yet been reported.

The transgenic OT-1 and gBT-I.1 CD8 ${ }^{+} \mathrm{T}$ cells featured in the present study are routinely used to assay antigen cross-presentation on MHC class I $(32,42)$. While this is a useful system, it is highly sensitive; hence, the data derived from this assay must be interpreted in the context that the $\mathrm{T}$ cell subsets being monitored in this model have receptors with single high-affinity antigen specificity. In this context, it is remarkable that even partial inhibition of efferocytosis via blockage of the AXL/LRP-1/ RANBP9 pathway resulted in inhibition of cross-presentation in this highly sensitive assay. It is likely that inhibition of crosspresentation to endogenous $T$ cells, which would presumably not express such high-affinity receptors, would be even higher than that observed with transgenic T cells. In support of this idea, our data demonstrated that blocking AXL/LRP-1/RANBP9-mediated efferocytosis resulted in enhanced HSV-1 pathogenesis, despite only approximately $30 \%$ inhibition in proliferation of gBT-I.1 transgenic T cells. Whether additional or alternative mechanisms are involved in this effect will require further study. For example, DNGR-1, a c-type lectin receptor that recognizes F-actin exposed by necrotic cells $(43,44)$, has been implicated in mediating crosspresentation and antiviral immunity by retaining the internalized necrotic cargo in an early endosomal compartment that facilitates cross-presentation $(45,46)$.
Efferocytosis prevents secondary necrosis of ACs, and defects in efferocytosis have been linked to pathological tissue necrosis, such as that in the necrotic cores of advanced, clinically dangerous atherosclerotic lesions (47). For example, deletion of the macrophage efferocytosis receptors MERTK and LRP-1 have been demonstrated to exacerbate necrotic core formation in mouse models of advanced atherosclerosis $(19,48,49)$. Interestingly, observational studies have shown that the necrotic cores of clinically dangerous human atheromata are often surrounded by mature DCs, which are relatively inefficient efferocytes that can constitute up to $30 \%$ of the phagocyte population of atherosclerotic lesions $(7,50,51)$. Thus, pathologic accumulation of pools of efferocytosis-inefficient mature DCs may promote focal necrosis. However, testing of this hypothesis in atherosclerosis and in other pathological settings, such as in tumor immunosurveillance, has been hampered by lack of knowledge of DC-specific efferocytosis receptors $(2,52)$. Here, we found that neither TIM-3 neutralization nor RANBP9 deficiency blocked efferocytosis by macrophages in vivo (Figure 2 and Supplemental Figure 6E); thus, targeting these molecules may provide a strategy by which to interrogate the role of DC-specific efferocytosis in these settings. Moreover, given the critical role of efferocytosis and antigen cross-presentation in suppressing autoimmune responses, future investigation of the role of the AXL/LRP-1/RANBP9 complex in this area is warranted.

In summary, we characterized a new multiprotein complex utilized by DCs for efferocytosis and antigen cross-presentation in vivo and demonstrated a novel role for the scaffolding protein RANBP9 in facilitating DC efferocytosis. In addition to providing new insight and tools with which to study the physiologic and pathologic roles of DC efferocytosis, these findings may have implications for future DC-based immunotherapeutic strategies. For example, proteins or peptides packaged in AC-mimetic nanoparticles targeted to the AXL/LRP-1/RANBP9 complex may enhance efficiency of antigen cross-presentation for the purpose of eliciting a desired immune response against cancer or pathogens or a tolerogenic response in the setting of autoimmunity.

\section{Methods}

Animals and animal maintenance. $\mathrm{Cd} 11 \mathrm{~b}^{-/-}$and $\mathrm{OT}-1$ mice were obtained from Jackson Laboratories (stock nos. 003991 and 003831, respectively). $A x \mathrm{l}^{\mathrm{H}^{-}}$ Tyro3 ${ }^{-/-}, A \mathrm{xl}^{-/-}$, and $\mathrm{Mertk}^{-/-}$mice were generated as described previously $(53,54)$. Lrp1 $1^{f / f l}$ mice (strain name B6;129S7-Lrp1tm2Her/J; JAX stock no. 012604) were generated as described previously (55) and then bred with $\mathrm{Cd} 11 \mathrm{cCre} \mathrm{C}^{+/+}$mice (Jackson Laboratories; stock no. 008068) to generate Cd11cCre $\operatorname{Lrp}^{1 / \mathrm{fl} / \mathrm{l}}$ mice. Ranbp $9^{-/-}$embryos were harvested from pregnant mice obtained from Ranbp $9^{+/-}$mating (27). Ranbp $9^{f / / f l}$ mice, with exon 3 flanked by lox $P$ sites, were generated using a targeting vector consisting of an FRT site-flanked $\beta$-neo cassette with loxP sites inserted upstream and downstream of exon 3 of the Ranbp9 gene. This construct was electrophoresed into JM8A3 F1-derived ES cells. An ES cell clone (HEPD0592.3.D06) that had successfully undergone FLP-mediated recombination and no longer retained the cassette, but did retain the loxP-flanked exon 3, was obtained from The European Conditional Mouse Mutagenesis Program and injected in to C57BL/ 6 blastocysts. Male chimeric mice were then bred to C57BL/6J female mice for germline transmission. Male and female heterozygous Ranbp $9^{f /+}$ mice were bred with each other to generate homozygous Ranbp $9^{f / f l}$ mice, which were used as a source of BMDCs for this study. gBT-I.1 mice were provided by D. Carr (University of Oklahoma Health Sciences Center, Oklahoma City, Oklahoma, USA). 
Primary culture of BMDCs. Murine bone marrow cells were isolated from the femurs of mice, cultured for 7 days in petri dishes in DMEM containing $10 \%$ FBS, and supplemented daily with GM-CSF (10 ng/ml) and IL-4 $(10 \mathrm{ng} / \mathrm{ml})$. On day 3 , half the medium was replaced to replenish nutrients. The nonadherent cells were harvested on day 7 , and experiments were performed by plating these cells on non-tissue culture-treated 24-well plates. To delete RANBP9 in BMDCs generated from the bone marrow of Ranbp $9^{f / f l}$ mice, cells were transduced with an adenoviral vector encoding Cre or LacZ control at an MOI of 200:1 (virus/DC), and experiments were conducted 48 hours later. Adeno-Cre and adeno-LacZ were obtained from Viraquest.

Generation of human peripheral blood monocyte-derived DCs. Buffy coats were obtained from New York Blood Center. The mononuclear cell layer was isolated by gradient centrifugation using Ficoll-Hypaque, then plated and incubated in tissue culture dishes. After 1 hour, the nonadherent cells were washed away. The medium (DMEM containing $10 \%$ FBS) was supplemented with 500 IU recombinant human GM-CSF and IL-4 on days 0 and 3 of culture. The nonadherent cell population on day 7 was used as DCs.

Human splenic DC isolation. Human spleen samples were maintained in cold saline and brought to the laboratory within 6 hours of organ procurement. In order to ensure high live cell yield, samples were rapidly processed through both enzymatic and mechanical digestion methods. Spleen was cleaned of fat, chopped into small pieces, and placed into 50-ml conical tubes. Enzymatic digestion media $(25 \mathrm{ml})$ was added (RPMI containing $10 \%$ FBS, L-glutamate, sodium pyruvate, nonessential amino acids, penicillin-streptomycin, $1 \mathrm{mg} / \mathrm{ml}$ collagenase $\mathrm{D}, 1 \mathrm{mg} / \mathrm{ml}$ trypsin inhibitor, and $0.1 \mathrm{mg} / \mathrm{ml}$ DNase I) and incubated with mechanical shaking at $37^{\circ} \mathrm{C}$ for 1 hour. Digested tissue was then disrupted using the gentleMACS tissue dissociator (Miltenyi Biotech), and the resulting suspension was passed through a stainless steel tissue sieve (mesh size, 10-150), followed by centrifugation. Red blood cell lysis was performed by addition of $5 \mathrm{ml} \mathrm{ACK}$ lysis buffer (Cellgro) directly to the cell pellet and incubation with periodic agitation for 5 minutes. To remove dead cells and debris, cell pellets were resuspended in $20 \mathrm{ml}$ of $30 \%$ percoll (GE Healthcare Life Sciences) and centrifuged at $50 \mathrm{~g}$ for 12 minutes. The resulting top layer and bottom pellet were discarded. Cells were washed with complete RPMI to remove percoll, and the resulting cell suspension was passed through a $40-\mu \mathrm{m}$ filter. DCs were obtained from this cell suspension by negative selection using human myeloid DC isolation kit (Miltenyi Biotech).

Generation of fluorescently labeled ACs. Mouse splenocytes (in vivo experiments) or Jurkat cells (in vitro experiments) were labeled with PKH67-GL ( $2 \mu \mathrm{M}$; Sigma-Aldrich) per the manufacturer's instructions. Cells were then exposed to ultraviolet light ( $254 \mathrm{~nm}$, UVP) for 5 minutes for induction of apoptosis. After UV exposure, cells were incubated in a $37^{\circ} \mathrm{C}$ incubator with $5 \% \mathrm{CO}_{2}$ for 1 hour (Jurkat cells) or 3 hours (splenocytes). We routinely obtained approximately $80 \%$ early $\mathrm{ACs}\left(\right.$ annexin $\mathrm{V}^{+} \mathrm{PI}-$ ) at these time points.

In vitro $A C$ binding and engulfment assay. AC binding experiments were conducted on BMDCs $\left(5 \times 10^{5}\right.$ cells/well on 24-well non-tissue culturecoated plates). Internalization was blocked either by pretreatment with $5 \mu \mathrm{g} / \mathrm{ml}$ cytochalasin D for 30 minutes, followed by incubation with PKH67-labeled ACs (1:5 BMDC/AC ratio) for 1 hour at $37^{\circ} \mathrm{C}$, or by incubation with ACs at $4^{\circ} \mathrm{C}$ for 1 hour. AC engulfment experiments were conducted by incubating DCs with ACs for 2 hours at $37^{\circ} \mathrm{C}$ in the absence of cytochalasin D. For quantification, cells were mounted on slides and analyzed by fluorescence microscopy to determine the percentage of DCs labeled with PKH67-tagged ACs.

In vivo splenic $D C$ efferocytosis assay. $2 \times 10^{7}$ apoptotic splenocytes were labeled ex vivo with $100 \mu \mathrm{g} / \mathrm{ml}$ PKH67-GL or Cypher-5E (GE Healthcare) per the manufacturer's instructions. The labeled cells were then injected i.v. into mice. For inhibition experiments, GST ( $2 \mathrm{mg} / \mathrm{mouse}$ i.v.), GST-RAP ( $2 \mathrm{mg} /$ mouse i.v.), TIM-3 nAb (100 $\mu \mathrm{g} /$ mouse i.p.), or appropriate IgG con- trol was injected into mice 30 minutes prior to injection of labeled ACs. 3 hours later, mice were euthanized by $\mathrm{CO}_{2}$ asphyxiation, and splenocytes were harvested by gently disaggregating the spleen tissue in a buffer containing $300 \mu \mathrm{g} / \mathrm{ml}$ collagenase D (Sigma-Aldrich). The splenocytes were stained with fluorophore-conjugated antibodies against $\mathrm{CD} 11 \mathrm{c}, \mathrm{CD} 8 \alpha$, and F4/80 (BD Biosciences) and analyzed by flow cytometry (BD FACS calibur or BD FACS canto II) using FlowJo software (Tree Star Inc.). Cell sorting was conducted at the flow cytometry core facility of Columbia Center for Translational Immunology using a BD influx cell sorter.

FRET assay. Antibodies against AXL and LRP-1 (R\&D Systems and Sigma-Aldrich, respectively) were conjugated to Alexa Fluor 546 or Alexa Fluor 647 using a commercially available antibody labeling kit (Invitrogen). The labeling ratio was approximately 3 , calculated according to the manufacturer's instructions. Antibody labeling of cells was performed at $4{ }^{\circ} \mathrm{C}$ for 30 minutes. FRET analysis was conducted by flow cytometry on CD $11 \mathrm{c}^{\text {hi }}$ gated cells using a BD FACS Calibur equipped with 488- and 635-nm lasers. Data were analyzed using FlowJo software.

$I P$ and Western blotting. IP was performed by lysing in vitro-cultured DCs using an IP buffer containing $20 \mathrm{mM}$ Tris- $\mathrm{HCl}$ ( $\mathrm{pH} 7.5), 150 \mathrm{mM} \mathrm{NaCl}$, $1 \mathrm{mM} \mathrm{MgCl}_{2}, 1 \mathrm{mM} \mathrm{CaCl}_{2}, 1 \%$ Triton X-100, and 1 tablet of Roche EDTAfree protease inhibitor per $10 \mathrm{ml}$ of buffer. Lysates were then incubated with protein A-sepharose beads (GE Healthcare) and $100 \mu \mathrm{g}$ anti-AXL (Cell Signaling Technology) or anti-LRP-1 (Sigma-Aldrich) antibody overnight at $4^{\circ} \mathrm{C}$ on a rotor-shaker. Samples were centrifuged at $90 \mathrm{~g}$ for 5 minutes, and beads were washed and recentrifuged 3 times with IP buffer. Laemmli buffer was then added to the beads; after boiling, the suspensions were centrifuged at $9,000 \mathrm{~g}$. The supernatant fractions were loaded onto $4 \%-20 \%$ Tris-glycine SDS-PAGE gels. After transfer onto nitrocellulose membranes, blots were probed for AXL (R\&D Systems), LRP-1 (Sigma-Aldrich), and RANBP9 (Epitomics) using specific antibodies, and the immunoreactive bands were visualized by enhanced chemiluminescence (Thermo Scientific).

siRNA transfection. BMDCs $\left(5 \times 10^{5}\right.$ cells/well of 24 -well non-tissue culture-treated plates) were resuspended in serum-free DMEM. siRNA transfection complex was prepared by incubating 20 pmol Gulp1 siRNA (Qiagen) with Lipofectamine 2000 (Invitrogen) (1:1 ratio) in serum-free DMEM for 20 minutes at room temperature. BMDCs were incubated with 20 pmol siRNA transfection complex under serum-free conditions for 6 hours at $37^{\circ} \mathrm{C}$ in a $5 \% \mathrm{CO}_{2}$ incubator, after which the culture medium was supplemented with serum to a final concentration of $5 \%$. Cells were harvested 24 hours after transfection; knockdown efficiency was analyzed by measuring Gulp1 mRNA by RT-qPCR.

Fetal liver transplantation. E15 embryos from pregnant mice of Ranbp $9^{+/-}$ matings were harvested. Portions of the tail and head were preserved for genotyping by PCR and Western blotting to identify Ranbp ${ }^{-/-}$embryos. Under sterile conditions, the fetal livers were harvested, and cell suspensions were prepared by passing the liver tissue sequentially 3 times through a 20-gauge needle and 1 time through a 25 -gauge needle. Cells were centrifuged at $130 \mathrm{~g}$ for 5 minutes, resuspended in serum-free DMEM, and counted using a hemocytometer. $2 \times 10^{6}$ cells were injected i.v. into lethally irradiated (10 Gy; Gammacell40 unit) C57BL/6J recipient mice. Recipient mice were fed antibiotic water $(100 \mu \mathrm{g} / \mathrm{ml}$ neomycin and $10 \mu \mathrm{g} / \mathrm{ml}$ polymyxin B) for 2 weeks and then acidified water. Chimeric mice were used 4 weeks after fetal liver transplantation.

In vitro antigen cross-presentation assay. Jurkat cells were incubated with $1 \mathrm{mg} / \mathrm{ml} \mathrm{OVA}$, and the OVA-loaded cells were exposed to UV light to induce apoptosis. BMDCs from appropriate groups of mice were incubated with OVA-loaded ACs for 2 hours to facilitate efferocytosis. FACS-sorted $\mathrm{CD}^{+} \mathrm{T}$ cells from OT-1 transgenic mice, which express TCR specific to OVA $257-264$, were labeled with $5 \mu \mathrm{M}$ CFSE as described previously (56). These CFSElabeled OT- $1 \mathrm{~T}$ cells $\left(5 \times 10^{4} /\right.$ well $)$ were then cultured with BMDCs 
exposed to OVA-loaded ACs $\left(10^{4} /\right.$ well) for 72 hours. Cells were stained with anti-CD8 $\alpha$ (BD Biosciences) to specifically detect OT-1 T cells. CFSE dye dilution as a readout of $\mathrm{T}$ cell proliferation was analyzed by flow cytometry on CD8 $\alpha^{+}$-gated cells.

HSV-1 culture, mouse infection, and virus titering. HSV-1 strain KOS was purchased from ATCC and propagated by infecting Vero cells (ATCC) as described previously (57). Mouse infection was conducted by i.v. injection of $4 \times 10^{5} \mathrm{pfu} / \mathrm{mouse}$ (self-limiting dose) or $2 \times 10^{8} \mathrm{pfu} / \mathrm{mouse}$ (lethal dose) of HSV-1 in an ABSL2 facility. For experiments assaying antigen crosspresentation, mice were also injected with $1 \times 10^{6}$ CFSE-labeled gBT-I.1 transgenic $\mathrm{CD}^{+} \mathrm{T}$ cells. Antigen cross-presentation was analyzed by measuring CFSE dye dilution on $\mathrm{CD}^{+} \mathrm{CFSE}^{+}$-gated cells on a flow cytometer. To estimate the virus burden in tissues of infected mice, homogenized tissue samples were sonicated, and the resultant supernatant fraction was used to conduct virus titer analysis. Briefly, supernatants at several dilutions were added to confluent Vero cells grown in $25-\mathrm{cm}^{2}$ tissue culture flasks and incubated for 2 hours. After washing away free virus, $7.5 \mu \mathrm{g} / \mathrm{ml}$ pooled human Ig (Sigma-Aldrich) was added to prevent reinfection via extracellular virus particles. The flasks were incubated at $37^{\circ} \mathrm{C}$ for 2 days, and then plaques were visualized by Giemsa staining and counted.

Primary adrenal cell culture and induction of apoptosis by HSV-1 infection. Primary adrenal cells were cultured as described previously (58). Briefly, mice were euthanized, and both adrenal glands were isolated, minced, and digested in HBSS containing $2 \mathrm{mg} / \mathrm{ml}$ collagenase I, $0.05 \mathrm{mg} / \mathrm{ml}$ DNase I, and $5 \mathrm{mg} / \mathrm{ml}$ bovine serum albumin. The isolated cells were cultured on tissue culture dishes in KO-DMEM (Invitrogen) supplemented with 15\% KO serum replacement (Invitrogen) for 3 days. To induce adrenal cell apoptosis, cells were infected with HSV-1 strain KOS (MOI, 10 pfu/cell) for 2 hours. Cells were further cultured for 24 hours (at which point annexin $\mathrm{V}$ labeling revealed $>65 \%$ of cells to be apoptotic), then UV-irradiated to inactivate the virus prior to injection in mice.

Statistics. All data are expressed as mean \pm SEM. Analysis of statistical significance was performed using 2-tailed Student's $t$ test; a $P$ value less than 0.05 was considered statistically significant.

1. Korns D, Frasch SC, Fernandez-Boyanapalli R, Henson PM, Bratton DL. Modulation of macrophage efferocytosis in inflammation. Front Immunol. 2011;2:57.

2. Thorp E, Subramanian M, Tabas I. The role of macrophages and dendritic cells in the clearance of apoptotic cells in advanced atherosclerosis. EurJ Immunol. 2011;41(9):2515-2518.

3. Scott RS, et al. Phagocytosis and clearance of apoptotic cells is mediated by MER. Nature. 2001;411(6834):207-211.

4. Cohen PL, et al. Delayed apoptotic cell clearance and lupus-like autoimmunity in mice lacking the c-mer membrane tyrosine kinase. J Exp Med. 2002;196(1):135-140.

5. Lemke G, Lu Q. Macrophage regulation by Tyro 3 family receptors. Curr Opin Immunol. 2003; 15(1):31-36.

6. Hanayama R, et al. Autoimmune disease and impaired uptake of apoptotic cells in MFG-E8deficient mice. Science. 2004;304(5674):1147-1150.

7. Albert ML, et al. Immature dendritic cells phagocytose apoptotic cells via $\alpha v \beta 5$ and CD36, and crosspresent antigens to cytotoxic T lymphocytes. J Exp Med. 1998;188(7):1359-1368.

8. Rock KL, Shen L. Cross-presentation: underlying mechanisms and role in immune surveillance. Immunol Rev. 2005;207:166-183.

9. Heath WR, Carbone FR. Cross-presentation in viral immunity and self-tolerance. Nat Rev Immunol. 2001;1(2):126-134.

10. Nakayama M, et al. Tim-3 mediates phagocytosis of apoptotic cells and cross-presentation. Blood.
Study approval. All animals used in this study were maintained in a barrier facility. All animal protocols were approved by the Columbia University Institutional Animal Care and Use Committee. Human spleens were obtained from deceased organ donors, at the time of organ acquisition for transplantation, through an approved research protocol and MTA with the New York Organ Donor Network (NYODN). All donors were 73 years or younger, were free of chronic disease and cancer, and were negative for hepatitis B and C and for HIV. Because tissue samples were obtained from deceased individuals lacking identifiers for our experiments, this study did not qualify as human subjects research, as confirmed by the Columbia University Institutional Review Board. Research consent for the donor tissue was obtained by the NYODN transplant coordinators.

\section{Acknowledgments}

We thank George Kuriakose for technical assistance with this project; Steven Burden (New York University, New York, New York, USA) for facilitating the import of $\operatorname{Lrp} 1^{f l / f l}$ mice; and Daniel Carr for providing gBT-I.1 mice. This work was supported by NIH, AHAF, The Consortium for Frontotemporal Dementia Research, and the Lupe Murchison Foundation (to J. Herz) as well as by NIH grants NMSS RG429 (to G.K. Matsushima), AI106697 (to D.L. Farber), AI101251 (to K. Liu), AG036859 (to M. Lakshmana), and HL106019, HL075662, and HL054591 (to I. Tabas).

Received for publication July 9, 2013, and accepted in revised form November 21, 2013.

Address correspondence to: Manikandan Subramanian, Columbia University, Department of Medicine, 630 West 168th Street, PH9406, New York, New York 10032, USA. Phone: 212.305.5669; Fax: 212.305.4834; E-mail: ms4144@columbia.edu. Or to: Ira Tabas, Columbia University, Department of Medicine, 630 West 168th Street, PH8E-105F, New York, New York 10032, USA. Phone: 212.305.9430; Fax: 212.305.4834; E-mail: iat1@columbia.edu.
2009;113(16):3821-3830.

11. Seitz HM, Camenisch TD, Lemke G, Earp HS, Matsushima GK. Macrophages and dendritic cells use different Axl/Mertk/Tyro3 receptors in clearance of apoptotic cells. J Immunol. 2007;178(9):5635-5642.

12. Schulz O, Pennington DJ, Hodivala-Dilke K, Febbraio $M$, Reis e Sousa C. CD 36 or $\alpha v \beta 3$ and $\alpha v \beta 5$ integrins are not essential for MHC class I cross-presentation of cell-associated antigen by $\mathrm{CD} 8 \alpha+$ murine dendritic cells. J Immunol. 2002;168(12):6057-6065.

13. Belz GT, et al. CD36 is differentially expressed by CD8+ splenic dendritic cells but is not required for cross-presentation in vivo. J Immunol. 2002; 168(12):6066-6070.

14. Sakuishi K, Jayaraman P, Behar SM, Anderson AC, Kuchroo VK. Emerging Tim-3 functions in antimicrobial and tumor immunity. Trends Immunol. 2011; 32(8):345-349.

15. Ramirez-Ortiz ZG, et al. The scavenger receptor SCARF1 mediates the clearance of apoptotic cells and prevents autoimmunity. Nat Immunol. 2013;14(9):917-926.

16. Morelli AE, et al. Internalization of circulating apoptotic cells by splenic marginal zone dendritic cells: dependence on complement receptors and effect on cytokine production. Blood. 2003;101(2):611-620.

17. Iyoda $\mathrm{T}$, et al. The CD8+ dendritic cell subset selectively endocytoses dying cells in culture and in vivo. J Exp Med. 2002;195(10):1289-1302.

18. Gardai SJ, et al. Cell-surface calreticulin initiates clearance of viable or apoptotic cells through transactivation of LRP on the phagocyte. Cell. 2005; 123(2):321-334.
19. Yancey PG, et al. Macrophage LRP-1 controls plaque cellularity by regulating efferocytosis and Akt activation. Arterioscl Thromb Vasc Biol. 2010;30(4):787-795.

20. Herz J, Goldstein JL, Strickland DK, Ho YK, Brown MS. 39-kDa protein modulates binding of ligands to low density lipoprotein receptor-related protein/alpha 2-macroglobulin receptor. J Biol Chem. 1991;266(31):21232-21238.

21. Qiu C-H, Miyake Y, Kaise H, Kitamura H, Ohara $\mathrm{O}$, Tanaka M. Novel subset of CD8 $\alpha+$ dendritic cells localized in the marginal zone is responsible for tolerance to cell-associated antigens. J Immunol. 2009;182(7):4127-4136.

22. Bachem A, et al. Superior antigen cross-presentation and XCR1 expression define human CD11 ${ }^{+}$ CD141+ cells as homologues of mouse CD8+ dendritic cells. J Exp Med. 2010;207(6):1273-1281.

23. Poulin LF, et al. Characterization of human DNGR-1+ BDCA3+ leukocytes as putative equivalents of mouse CD8alpha+ dendritic cells. J Exp Med. 2010;207(6):1261-1271.

24. Hoffmann PR, et al. Phosphatidylserine (PS) induces PS receptor-mediated macropinocytosis and promotes clearance of apoptotic cells. J Cell Biol. 2001;155(4):649-660.

25. Hafizi S, Gustafsson A, Stenhoff J, Dahlbäck B. The Ran binding protein RanBPM interacts with Axl and Sky receptor tyrosine kinases. Int J Biochem Cell Biol. 2005;37(11):2344-2356.

26. Lakshmana MK, Yoon I-S, Chen E, Bianchi E, Koo EH, Kang DE. Novel role of RanBP9 in BACE1 processing of amyloid precursor protein and amyloid $\beta$ peptide generation. J Biol Chem. 
2009;284(18):11863-11872.

27. Lakshmana MK, et al. Role of RanBP9 on amyloidogenic processing of APP and synaptic protein levels in the mouse brain. FASEB J.2012;26(5):2072-2083.

28. Kinchen JM, et al. Two pathways converge at CED-10 to mediate actin rearrangement and corpse removal in C. elegans. Nature. 2005;434(7029):93-99.

29. Su HP, et al. Interaction of CED-6/GULP, an adapter protein involved in engulfment of apoptotic cells with CED-1 and CD91/Low density lipoprotein receptor-related protein (LRP). J Biol Chem. 2002; 277(14):11772-11779.

30. Ma Z, Nie Z, Luo R, Ravichandran KS. Regulation of Arf6 and ACAP1 signaling by the PTB-domaincontaining adaptor protein GULP. Curr Biol. 2007; 17(8):722-727.

31. Jirmo AC, Nagel C-H, Bohnen C, Sodeik B, Behrens GMN. Contribution of direct and cross-presentation to CTL immunity against Herpes simplex virus 1. J Immunol. 2009;182(1):283-292.

32. Mueller SN, Heath W, McLain JD, Carbone FR, Jones CM. Characterization of two TCR transgenic mouse lines specific for herpes simplex virus. Immunol Cell Biol. 2002;80(2):156-163.

33. Aita K, Irie H, Koyama AH, Fukuda A, Yoshida T, Shiga J. Acute adrenal infection by HSV-1: role of apoptosis in viral replication. Arch Virol. 2001; 146(10):2009-2020.

34. Bhattacharyya S, et al. Enveloped viruses disable innate immune responses in dendritic cells by direct activation of TAM receptors. Cell Host Microbe. 2013;14(2):136-147.

35. Erwig LP, Henson PM. Clearance of apoptotic cells by phagocytes. Cell Death Differ. 2007;15(2):243-250.

36. Tacken PJ, de Vries IJM, Torensma R, Figdor CG. Dendritic-cell immunotherapy: from ex vivo loading to in vivo targeting. Nat Rev Immunol. 2007; 7(10):790-802

37. Ishimoto Y, Ohashi K, Mizuno K, Nakano T. Promotion of the uptake of PS liposomes and apoptotic cells by a product of growth arrest-specific gene, gas6. J Biochem. 2000;127(3):411-417.

38. Albert ML, Sauter B, Bhardwaj N. Dendritic cells acquire antigen from apoptotic cells and induce class Irestricted CTLs. Nature. 1998;392(6671):86-89.

39. Bosnjak L, Miranda-Saksena M, Koelle DM, Boadle RA, Jones CA, Cunningham AL. Herpes simplex virus infection of human dendritic cells induces apoptosis and allows cross-presentation via uninfected dendritic cells. JImmunol. 2005;174(4):2220-2227.

40. Joffre OP, Segura E, Savina A, Amigorena S. Crosspresentation by dendritic cells. Nat Rev Immunol. 2012;12(8):557-569.

41. Chatterjee B, et al. Internalization and endosomal degradation of receptor-bound antigens regulate the efficiency of cross presentation by human dendritic cells. Blood. 2012;120(10):2011-2020.

42. Hogquist KA, Jameson SC, Heath WR, Howard JL, Bevan MJ, Carbone FR. T cell receptor antagonist peptides induce positive selection. Cell. 1994; 76(1):17-27.

43. Ahrens $\mathrm{S}$, et al. F-Actin is an evolutionarily conserved damage-associated molecular pattern recognized by DNGR-1, a receptor for dead cells. Immunity. 2012;36(4):635-645.

44. Zhang J-G, et al. The dendritic cell receptor Clec9A binds damaged cells via exposed actin filaments. Immunity. 2012;36(4):646-657.

45. Iborra S, Izquierdo HM, Martínez-López M, BlancoMenéndez N, Reis e Sousa C, Sancho D. The DC receptor DNGR-1 mediates cross-priming of CTLs during vaccinia virus infection in mice. J Clin Invest. 2012;122(5):1628-1643

46. Zelenay S, et al. The dendritic cell receptor DNGR1 controls endocytic handling of necrotic cell antigens to favor cross-priming of CTLs in virusinfected mice. J Clin Invest. 2012;122(5):1615-1627.

47. Tabas I. Macrophage death and defective inflammation resolution in atherosclerosis. Nat Rev Immunol. 2010;10(1):36-46.

48. Thorp E, Cui D, Schrijvers DM, Kuriakose G, Tabas I. Mertk receptor mutation reduces efferocytosis efficiency and promotes apoptotic cell accumulation and plaque necrosis in atherosclerotic lesions of Apoe ${ }^{-/-}$mice. Arterioscl Thromb Vasc Biol. 2008; 28(8):1421-1428

49. Ait-Oufella H, et al. Defective Mer receptor tyrosine kinase signaling in bone marrow cells promotes apoptotic cell accumulation and accelerates atherosclerosis. Arterioscl Thromb Vasc Biol. 2008; 28(8):1429-1431.

50. Yilmaz A, et al. Emergence of dendritic cells in rupture-prone regions of vulnerable carotid plaques. Atherosclerosis. 2004;176(1):101-110.

51. Christ A, Eijgelaar W, Daemen M. The role of dendritic cells in human atherosclerotic lesions. Cardiovasc Res. 2010;87:S129.

52. Rock KL, Shen L. Cross-presentation: underlying mechanisms and role in immune surveillance. Immunol Rev. 2005;207:166-183.

53. Camenisch TD, Koller BH, Earp HS, Matsushima GK. A novel receptor tyrosine kinase, Mer, inhibits TNF- $\alpha$ production and lipopolysaccharide-induced endotoxic shock. J Immunol. 1999;162(6):3498-3503.

54. Williams JC, Wagner NJ, Earp HS, Vilen BJ, Matsushima GK. Increased hematopoietic cells in the $\mathrm{Mertk}^{-/}$ mouse peritoneal cavity: A result of augmented migration. J Immunol. 2010;184(12):6637-6648.

55. Rohlmann A, Gotthardt M, Willnow TE, Hammer RE, Herz J. Sustained somatic gene inactivation by viral transfer of Cre recombinase. Nat Biotech. 1996;14(11):1562-1565.

56. Quah BJC, Warren HS, Parish CR. Monitoring lymphocyte proliferation in vitro and in vivo with the intracellular fluorescent dye carboxyfluorescein diacetate succinimidyl ester. Nat Protoc. 2007; 2(9):2049-2056

57. Blaho JA, Morton ER, Yedowitz JC. Herpes simplex virus: Propagation, quantification, and storage. Curr Protoc Microbiol. 2005; Chapter 14:Unit 14E.1.

58. Chu Y, Wu BM, McCabe ER, Dunn JCY. Serum-free cultures of murine adrenal cortical cells. J Pediatr Surg. 2006;41(12):2008-2012. 